NASA/TM-2002-211415

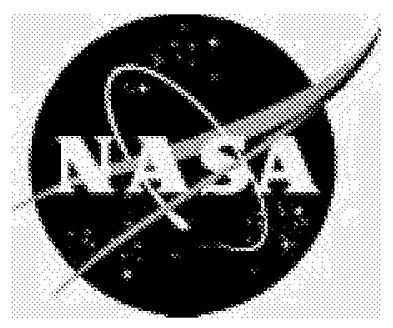

\title{
On Accuracy of Adaptive Grid Methods for Captured Shocks
}

Nail K. Yamaleev and Mark H. Carpenter

Langley Research Center, Hampton, Virginia 


\section{The NASA STI Program Office ... in Profile}

Since its founding, NASA has been dedicated to the advancement of aeronautics and space science. The NASA Scientific and Technical Information (STI) Program Office plays a key part in helping NASA maintain this important role.

The NASA STI Program Office is operated by Langley Research Center, the lead center for NASA's scientific and technical information. The NASA STI Program Office provides access to the NASA STI Database, the largest collection of aeronautical and space science STI in the world. The Program Office is also NASA's institutional mechanism for disseminating the results of its research and development activities. These results are published by NASA in the NASA STI Report Series, which includes the following report types:

- TECHNICAL PUBLICATION. Reports of completed research or a major significant phase of research that present the results of NASA programs and include extensive data or theoretical analysis. Includes compilations of significant scientific and technical data and information deemed to be of continuing reference value. NASA counterpart of peer-reviewed formal professional papers, but having less stringent limitations on manuscript length and extent of graphic presentations.

- TECHNICAL MEMORANDUM. Scientific and technical findings that are preliminary or of specialized interest, e.g., quick release reports, working papers, and bibliographies that contain minimal annotation. Does not contain extensive analysis.

- CONTRACTOR REPORT. Scientific and technical findings by NASA-sponsored contractors and grantees.
- CONFERENCE PUBLICATION Collected papers from scientific and technical conferences, symposia, seminars, or other meetings sponsored or co-sponsored by NASA.

- SPECIAL PUBLICATION. Scientific, technical, or historical information from NASA programs, projects, and missions, often concerned with subjects having substantial public interest.

- TECHNICAL TRANSLATION. Englishlanguage translations of foreign scientific and technical material pertinent to NASA's mission.

Specialized services that complement the STI Program Office's diverse offerings include creating custom thesauri, building customized databases, organizing and publishing research results ... even providing videos.

For more information about the NASA STI Program Office, see the following:

- Access the NASA STI Program Home Page at http://www.sti.nasa.gov

- E-mail your question via the Internet to help@sti.nasa.gov

- Fax your question to the NASA STI Help Desk at (301) 621-0134

- Phone the NASA STI Help Desk at (301) 621-0390

- Write to: NASA STI Help Desk NASA Center for AeroSpace Information 7121 Standard Drive Hanover, MD 21076-1320 
NASA/TM-2002-211415

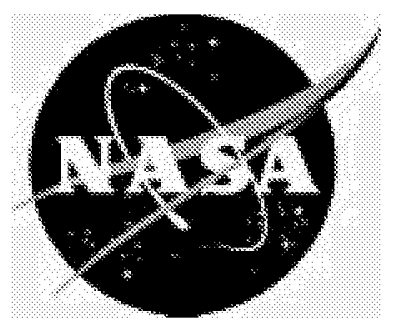

\section{On Accuracy of Adaptive Grid Methods for Captured Shocks}

Nail K. Yamaleev and Mark H. Carpenter

Langley Research Center, Hampton, Virginia

National Aeronautics and

Space Administration

Langley Research Center

Hampton, Virginia 23681-2199 
Available from:

NASA Center for AeroSpace Information (CASI)

7121 Standard Drive

Hanover, MD 21076-1320

(301) 621-0390
National Technical Information Service (NTIS)

5285 Port Royal Road

Springfield, VA 22161-2171

(703) 605-6000 


\title{
On Accuracy of Adaptive Grid Methods for Captured Shocks
}

\author{
Nail K. Yamaleev ${ }^{\dagger}$ and Mark H. Carpenter ${ }^{\ddagger}$ \\ $\dagger$ National Research Council, USA;

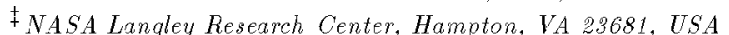 \\ E-mail: nail@tabdemo.larc.nasa.gov; m.h.carpenter@larc.nasa.gov
}

\begin{abstract}
The accuracy of two grid adaptation stratcgics, grid redistribution and local grid refincment, is cxamincd by solving the 2-D Eulcr cquations for the supcrsonic stcady flow around a cylinder. Sccond- and fourth-order lincar finitc difference shock-capturing schemes, bascd on the Lax-Fricdrichs flux splitting, are uscd to discretizc the governing cquations. The grid refincment study shows that for the second-order scheme, ncither grid adaptation strategy improves the numcrical solution accuracy compared to that calculatcd on a uniform grid with the same number of grid points. For the fourth-order scheme, the dominant first-order error component is reduced by the grid adaptation, whilc the design-order crror component drastically incrcases because of the grid nonuniformity. As a result, both grid adaptation techniques improve the numcrical solution accuracy only on the coarscst mcsh or on very finc grids that arc scldom found in practical applications becausc of the computational cost involved. Similar crror bchavior has becn obtaincd for the prcssurc intcgral across the shock. $\Lambda$ simple analysis shows that both grid adaptation stratcgics are not without penaltics in the numcrical solution accuracy. Bascd on thesc results, a ncw grid adaptation critcrion for capturcd shocks is proposcd.
\end{abstract}

Kcy Words: high-order accuracy, grid adaptation, local grid refincment, grid redistribution, shock capturing, finite difference scheme.

\section{INTRODUCTION}

Wave propagation phenomena in computational fluid dynamics, computational aeroacoustics, computational electromagnetics, and large eddy or direct numerical simulation of turbulence are characterized by the presence of both a lot of structure in the smooth part of the solution and strong discontinuities. For this class of problems, attaining the design accuracy of high-order shock-capturing methods is problematic. $\Lambda$ s has recently been shown for 1-D unsteady and 2-D steady shocked flows, the first-order error introduced by shock capturing can persist globally down- 
stream $[1,2]$. The result is that the numerical solution is just first-order accurate downstream of the discontinuity, regardless of the design accuracy of the discretization used. Similar degeneration in accuracy for captured discontinuities has also been reported in [3].

One way of removing the first-order error component from the numerical solution is shock fitting. $\Lambda$ s shown in $[1,2]$, if a cell interface is aligned with the shock, and a high-order conservative essentially nonoscillatory (ENO) formulation based on a Roe flux which satisfies the Rankine-Hugoniot shock jump relations is used to calculate the interface fluxes, then the design order of accuracy can be recovered. Despite its simplicity, this method has serious disadvantages. Detection and localization of multidimensional complex shocks, generalization of this approach to moving and interacting shocks, and use of ENO type approximations in singular regions where the smooth interpolants cannot be constructed make this approach unreliable, and, therefore, little used in real numerical applications.

An alternative strategy is to reduce grid spacing locally near a shock rather than refine the grid globally. The idea of this method is based on an assumption that the first-order error is generated locally at the shock and then transported downstream. This approach, known as a grid adaptation, has been extensively used to improve resolution of captured discontinuities for at least twenty years, e.g., see [4]. There are two basic strategies of grid adaptation: local grid refinement and grid redistribution. In the first approach, grid nodes are added to locally enrich the grid to achieve higher accuracy. In the second approach, the number of grid cells is fixed and the position of grid points is adjusted to improve the numerical solution accuracy. Until now, little attention has been paid to one of the most important problems associated with the adaptive grid methods: the essential effect of the grid points distribution on error in the numerical solution. It should be emphasized that concentration of grid points in regions which most influence the numerical solution accuracy may at the same time introduce additional error because of the grid nonuniformity [5].

Most adaptive grid methods are based on the error equidistribution principle developed in [6], [7], [8], which, in turn, is driven by one or another error estimation technique. One of the widely used error estimators is gradient or local curvature of the numerical solution $[9,10,11]$. An alternative method is to equidistribute the local truncation error estimate or the finite element residual which is similar to the finite difference truncation error $[12,13]$. Another class of error estimators is based on evaluating the solution interpolation error [14]. For second-order discretizations, this method is reduced to estimation of the local curvature of the numerical solution. Richardson extrapolation is also used to estimate error in the numerical solution [15]. This procedure compares the solution obtained on the existing grid with one computed on a grid that is twice as coarse in each spatial direction.

Although the error estimators mentioned above are quite different, all of them rely on certain smoothness of the differential solution that is not the case for discontinuous flows. In fact, most grid adaptation criteria that can be found in the literature become singular at discontinuities. To remove this singularity and to make the adaptive grid sufficiently smooth, a grid smoothing procedure must be used. As a result, the grid adaptation near discontinuities is driven by the grid smoothing procedure rather than the error estimate itself. 
If grid cell interfaces are not aligned with a shock, the captured discontinuity is always smeared over several grid points, which leads to the $O(1)$ error near the shock. Since the numerical solution is first-order accurate away from the shock, the true error and its estimates achieve their maximum values at the discontinuity. $\Lambda$ s a result, any grid adaptation procedure based on either the true error or its estimate leads to excessive clustering of grid points or local grid refinement near the shock. This kind of a grid adaptation is intended to reduce the solution error in the vicinity of discontinuities, but it does not necessarily guarantee improvement in accuracy in regions where the solution is smooth. As shown in [16], the conventional adaptive mesh refinement procedure based on gradient or local curvature of the numerical solution can lead to large error in the shock location caused by insufficient accuracy in smooth regions of flow ahead of and behind the discontinuity.

In the present paper, the accuracy of the grid redistribution and local grid refinement methods are studied. The 2-D test problem used is the supersonic flow around a circular cylinder, for which a Chebyshev bow-shock fitting spectral method is employed to obtain a very accurate numerical solution [17]. This solution is used as the "exact" solution in all subsequent refinement studies. The Euler equations are approximated with second- and fourth-order linear shock-capturing schemes based on the Lax-Friedrichs splitting of the flux vector. The refinement studies show that for the second-order scheme, neither grid adaptation strategy improves the numerical solution accuracy compared to that calculated on a uniform grid with the same number of grid points. For the fourth-order scheme, the dominant first-order error component is reduced by the grid adaptation, while the high-order error component drastically increases, because of the grid nonuniformity. $\Lambda$ s a result, the grid adaptation provides improvement in the solution accuracy only asymptotically. $\Lambda$ simple error analysis of the grid redistribution and local grid refinement methods reveals the main reasons why the grid adaptation methods do not improve the accuracy of captured discontinuities. Summarizing the numerical and theoretical results, we propose a new grid adaptation criterion for captured discontinuities.

The paper is organized as follows. Section 2 presents the blunt body problem and the spectral solution. Section 3 presents the finite difference methods used in the studies. Section 4 presents the grid adaptation strategies, including grid redistribution and local grid refinement. Section 5 presents the error analysis for the effects of grid adaptation on solution accuracy. Section 6 presents the secondand fourth-order results, Section 7 presents the new grid adaptation criterion for captured shocks, and Section 8 presents the conclusions of the work.

\section{SETTING OF A PROBLEM AND ITS "EXACT" SOLUTION}

We consider the steady-state supersonic inviscid gas flow around a circular cylinder. This test problem is desirable because the bow shock is 2-D, rather than simply a 1-D shock rotated into a 2-D reference frame. Furthermore, the problem is simple enough to obtain an "exact" solution.

The conservation law form of the 2-D Euler equations written in Cartesian coordinates $(x, y)$, as follows:

$$
\frac{\partial \mathbf{U}}{\partial t}+\frac{\partial \mathbf{F}}{\partial x}+\frac{\partial \mathbf{G}}{\partial y}=0,
$$




$$
\mathbf{U}=\left[\begin{array}{c}
\rho \\
\rho u \\
\rho v \\
\rho e
\end{array}\right], \mathbf{F}=\left[\begin{array}{c}
\rho u \\
\rho u^{2}+P \\
\rho v u \\
u(\rho e+P)
\end{array}\right], \mathbf{G}=\left[\begin{array}{c}
\rho v \\
\rho v u \\
\rho v^{2}+P \\
v(\rho e+P)
\end{array}\right],
$$

is used to describe the flowfield. The variables $\rho, u, v, P$, and $e$ are the density, $x-$ velocity, $y$-velocity, pressure, and total specific energy, respectively. The governing equations are closed with the equation of state for a perfect gas

$$
P=(\gamma-1) \rho\left[e-\frac{1}{2}\left(u^{2}+v^{2}\right)\right]
$$

where $\gamma$ is the ratio of specific heats, which is assumed to have a constant value of 1.4 .

To use different grid adaptation techniques, a differentiable one-to-one coordinate transformation,

$$
\begin{aligned}
& \tau=t \\
& \xi=\xi(x, y) \\
& \eta=\eta(x, y),
\end{aligned}
$$

is applied to map a physical domain with curvilinear boundaries onto a unit square. Note that the $\xi$ and $\eta$ coordinates do not depend on time and, therefore, moving grids are not considered in the present study.

The Euler equations in the curvilinear coordinates (2) can be written in conservation law form as

$$
\begin{gathered}
\frac{\partial \hat{\mathbf{U}}}{\partial \tau}+\frac{\partial \hat{\mathbf{F}}}{\partial \xi}+\frac{\partial \hat{\mathbf{G}}}{\partial \eta}=0, \\
\hat{\mathbf{U}}=\frac{1}{J} \mathbf{U}, \quad \hat{\mathbf{F}}=\frac{1}{J}\left(\xi_{x} \mathbf{F}+\xi_{y} \mathbf{G}\right), \quad \hat{\mathbf{G}}=\frac{1}{J}\left(\eta_{x} \mathbf{F}+\eta_{y} \mathbf{G}\right),
\end{gathered}
$$

where the Jacobian of the mapping is given by

$$
J=\frac{\partial(\xi, \eta)}{\partial(x, y)}=\xi_{x} \eta_{y}-\xi_{y} \eta_{x}
$$

To close the governing equations, boundary conditions should be specified. Because of the symmetry along the body centerline, only half of the domain is considered. The following boundary conditions are imposed along the symmetry line:

$$
\left.v\right|_{\eta=0}=0,\left.\quad \frac{\partial u}{\partial \eta}\right|_{\eta=0}=\left.\frac{\partial P}{\partial \eta}\right|_{\eta=0}=\left.\frac{\partial \rho}{\partial \eta}\right|_{\eta=0}=0 .
$$

On the cylinder surface, the no penetration boundary condition,

$$
u y_{\xi}-\left.v x_{\xi}\right|_{\xi=1}=0
$$

is imposed. $\Lambda$ t the supersonic inflow, all flow quantities are prescribed. The outflow boundary is chosen so that the outflow is fully supersonic and, therefore, no boundary conditions are imposed. 
$\Lambda$ spectrally accurate numerical solution to the blunt body problem described above is found by using a Chebyshev bow-shock fitting algorithm [17]. The shock position always coincides with the inflow boundary along which the RankineHugoniot relations are used. The physical domain changes continuously as the bow shock moves to its steady state position, while the computational domain remains unchanged. The Chebyshev collocation method is employed in both the radial and circumferential directions to discretize the Euler equations. The equations are then marched in time until a steady state solution is reached. Further details on the Chebyshev shock-fitting technique can be found in [17]. $\Lambda$ s has been shown in [2], the spectral solution is exact to at least eight significant digits. This solution, which is further referred to as the "exact" solution, is spectrally interpolated to a sequence of uniformly spaced grids to evaluate error in finite difference solutions.

\section{SECOND- AND FOURTH-ORDER NUMERICAL METHODS}

Second-order fully upwind and fourth-order upwind-biased linear finite difference schemes based on the Lax-Friedrichs flux splitting are used to discretize the Euler equations. These approximations can be written in a semi-discrete form as

$$
\frac{d \hat{\mathbf{U}}}{d \tau}=\frac{1}{2}\left(D_{\xi}^{-} \hat{\mathbf{F}}^{+}+D_{\xi}^{+} \hat{\mathbf{F}}^{-}\right)+\frac{1}{2}\left(D_{\eta}^{-} \hat{\mathbf{G}}^{+}+D_{\eta}^{+} \hat{\mathbf{G}}^{-}\right),
$$

where $D_{\xi}^{ \pm}$and $D_{\eta}^{ \pm}$are linear finite difference operators in $\xi$ and $\eta$, respectively. The Lax-Friedrichs fluxes are given by

$$
\begin{aligned}
\hat{\mathbf{F}}^{ \pm} & =\hat{\mathbf{F}} \pm\left|\lambda_{\xi}^{\max }\right| \hat{\mathbf{U}} \\
\hat{\mathbf{G}}^{ \pm} & =\hat{\mathbf{G}} \pm\left|\lambda_{\eta}^{\max }\right| \hat{\mathbf{U}}
\end{aligned}
$$

where $\left|\lambda_{\xi}^{\max }\right|$ and $\left|\lambda_{\eta}^{\max }\right|$ are the maximum values over the entire domain of the contravariant eigenvalues $|\hat{u}|+\hat{c}$ and $|\hat{v}|+\hat{c}$, accordingly.

The following second- and fourth-order spatial operators, $D_{2}^{ \pm}$and $D_{4}^{ \pm}$, are used in the present analysis:

$$
\begin{gathered}
D_{2}^{-} u_{j}=\frac{1}{2 h}\left(u_{j-2}-4 u_{j-1}+3 u_{j}\right) \\
D_{2}^{+} u_{j}=\frac{1}{2 h}\left(-3 u_{j}+4 u_{j+1}-u_{j+2}\right), \\
D_{4}^{-} u_{j}=\frac{1}{12 h}\left(-u_{j-3}+6 u_{j-2}-18 u_{j-1}+10 u_{j}+3 u_{j+1}\right) \\
D_{4}^{+} u_{j}=\frac{1}{12 h}\left(-3 u_{j-1}-10 u_{j}+18 u_{j+1}-6 u_{j+2}+u_{j+3}\right),
\end{gathered}
$$

where $h$ is a grid spacing either in $\xi$ or in $\eta$. For the second-order scheme (8), the second-order boundary closure is employed at all boundary points. For the fourthorder scheme, the third-order stencils used near the boundaries are the optimal stencils derived from nearest neighbor information, biased where possible in an upwind direction. These second- and fourth-order methods will be referred to as "LF-2-2" and "LF-4-3", respectively.

All finite difference grids considered in the present study are constructed such that a corner grid point coincides with the stagnation point. It has been found that this finite difference formulation may lead to numerical instability if all the boundary conditions on the symmetry line are imposed weakly through the flux. 
This instability is caused by the weak formulation of the boundary condition for the $v$ component of the velocity vector. Imposing this boundary condition in the strong sense eliminates the numerical instability. Numerical calculations have shown that the present formulation is as robust as the staggered formulation typically adopted by finite difference algorithms near stagnation points.

On the supersonic inflow, the entire state vector $\left.\mathbf{U}\right|_{\xi=0}$ is specified. On the outflow boundary, the solution is calculated by using high-order fully upwind approximations, i.e., no boundary conditions are imposed, which is consistent with the characteristic analysis for the supersonic outflow. On the impermeable wall, the no penetration boundary condition is imposed weakly by solving the Riemann problem approximately. Note that at steady state, the normal velocity at the wall is nonzero, but converges to zero with an order property consistent with overall formulation.

$\Lambda$ three stage explicit Runge-Kutta method is used to drive the solution to steadystate. To accelerate the convergence, the implicit residual smoothing method proposed in [18] is employed.

\section{GRID ADAPTATION METHODS}

The main purpose of the present study is to evaluate the influences of grid adaptation on overall solution accuracy, in the presence of the first-order error component resulting from shock capturing. The grid refinement studies presented in $[1,2]$ show that high-order shock-capturing schemes are first-order accurate downstream of shocks. Hence, away from the shock, the pointwise error is proportional to the grid spacing, and consequently decays like $O(h)$ as the grid is refined. $\Lambda t$ the same time, if a grid is not aligned with the discontinuity, any shock-capturing numerical scheme gives a discrete shock profile with at least one intermediate point in the shock. Thus, on sufficiently fine grids, the true pointwise error achieves its global maximum value of $O(1)$ at the discontinuity. To demonstrate this error behavior, the centerline pressure error distribution for the $M_{\infty}=3$ blunt body problem calculated using the LF-4-3 scheme is shown in Fig. 1. As one can see in the figure, the true pointwise error is singular at the shock located at $x=1.698$. This conclusion is based on the property of shock-capturing schemes and does not depend on grid spacing in the vicinity of the shock. As a result, any grid adaptation procedure based on the error equidistribution principle and the true error will always concentrate grid points or refine the grid near discontinuities of the solution.

It should be emphasized that the error equidistribution principle is not valid for problems with strong discontinuities. Actually, this principle is based on the minimization of the integral norm of the solution error or its estimate, which, in turn, is obtained as the solution of the Euler-Lagrange differential equation which cannot be used for discontinuous functions.

Furthermore, all error estimators found in the literature, such as estimators based on the truncation error, the finite element residual, recovery techniques, and the extrapolation, require certain smoothness of the solution of the original differential problem, which is not a property of discontinuous flows. $\Lambda$ s a result, these error estimators are singular in regions where the solution is discontinuous. This singularity corresponding to the singularity of the true error at the shock leads to excessive grid refinement or clustering of grid points around the shock. Asymptotically, any 


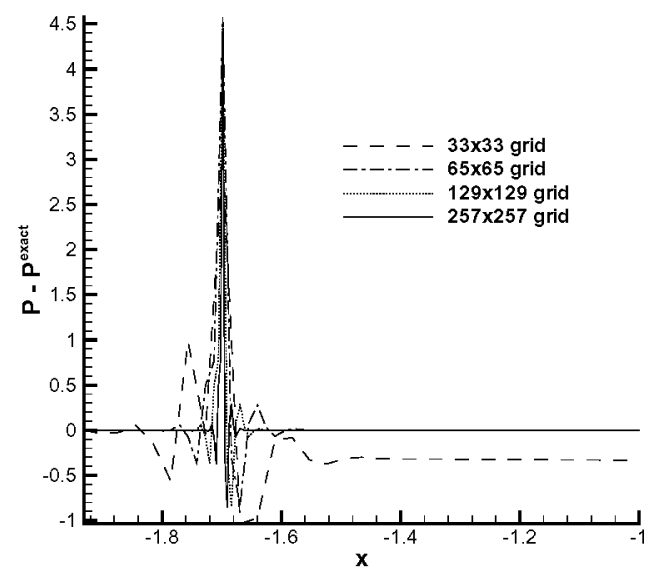

FIG. 1. Pointwise error for the $M_{\infty}=3$ blunt body problem obtained with the LF-4-3 scheme on uniform grids.

grid generator based on the error equidistribution principle and one of the error estimation procedures mentioned above would generate an adaptive grid such that the ratio of the local grid spacing at the shock to the neighboring one, generated in regions where the solution is smooth, approaches zero. This degeneration in grid spacing occurs because these error estimators become singular at the discontinuity. In practical applications, this singularity is eliminated by excessive smoothing of the error estimate function. $\Lambda$ s a result, the grid adaptation in the vicinity of the shock is driven by the smoothing procedure rather than by the error estimate itself. Therefore, instead of considering different error estimators and different grid smoothing techniques, we generate $C^{\infty}$ adaptive grids which are clustered or locally refined near the shock. This kind of a grid adaptation is intended to reduce the first-order error component caused by the shock-capturing procedure and to increase the overall solution accuracy towards the design accuracy of the numerical scheme used.

Because we know the exact solution, and consequently the exact shock location, an adaptive grid can be generated analytically. Quasi-one-dimensional grid adaptation can be employed, assuming that one family of grid lines is aligned with the bow shock. It can be done because the numerical solution error in the circumferential direction, which is aligned with the shock, is much smaller than that in the radial direction. To demonstrate this property, we solve the blunt body problem at $M_{\infty}=3$ by using the LF-4-3 scheme on two sequences of uniform grids. The first one is obtained by doubling the number of grid points in both the radial and circumferential directions, as follows: $33 \times 33,65 \times 65,129 \times 129$, and $257 \times 257$. The second one is generated by refining the grid only in the radial direction, while the number of grid points in the circumferential direction remains fixed and equal to 33 for all the grids considered. Figure 2 shows that the numerical solution errors obtained on these grids are practically identical. It indicates that the discretization error in the direction parallel to the shock is several orders of magnitude less 


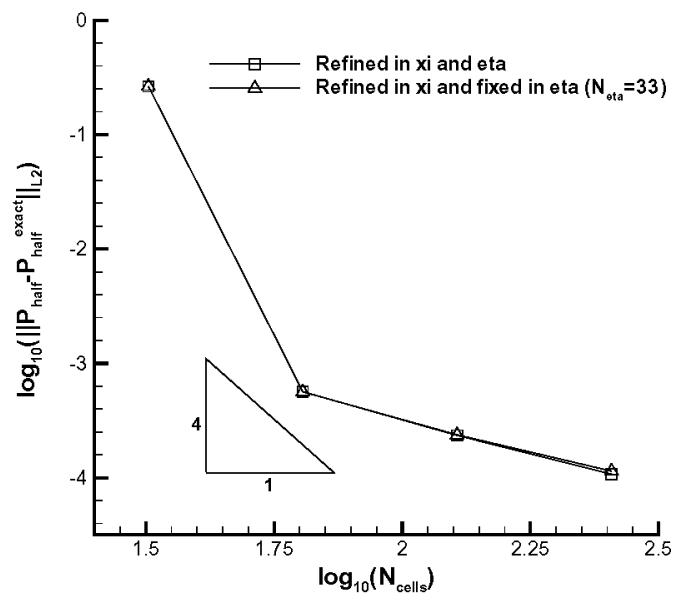

FIG. 2. Grid refinement study at $M_{\infty}=3$, showing the pressure error obtained with the LF-4-3 scheme on two sequences of grids: 1) uniformly refined in $\xi$ and $\eta$, and 2) refined in $\xi$, while the number of grid points in $\eta$ is fixed and equal to 33.

than that in the radial direction. Since the numerical solution error is strongly dominated by the radial error component, we use 1-D grid adaptation with the grid points constrained to move along one family of fixed radial coordinate lines, such that the grid is always aligned with the exact bow shock. Note that this grid adaptation procedure does not produce skewed cells where the solution accuracy may deteriorate because of small values of the Jacobian [4].

\subsection{Grid Redistribution Method}

Uniform meshes employed for finite difference calculations are constructed by using polar coordinatcs:

$$
\begin{aligned}
& x=-r \cos \theta \\
& y=r \sin \theta .
\end{aligned}
$$

$\Lambda$ mapping from the polar to the computational coordinates is given by

$$
\begin{aligned}
& \xi=1-\frac{r-r_{1}}{r_{0}(\theta)-r 1} \\
& \eta=\theta / \theta_{\max },
\end{aligned}
$$

where $r_{0}, r_{1}$, and $\theta_{\max }$ are the radius of the inflow boundary (which, in the case of the exact solution, coincides with the bow shock), the radius of the cylinder, and the maximum value of $\theta$, respectively. Note that the inflow boundary has been chosen so that the bow shock standoff distance is equal to $3 / 4\left(r_{0}(\theta)-r_{1}\right)$ for all meshes considered.

$\Lambda$ s mentioned above, an adaptive grid is constructed by redistributing grid points in 1-D fashion along the fixed radial lines. The following coordinate transformation 


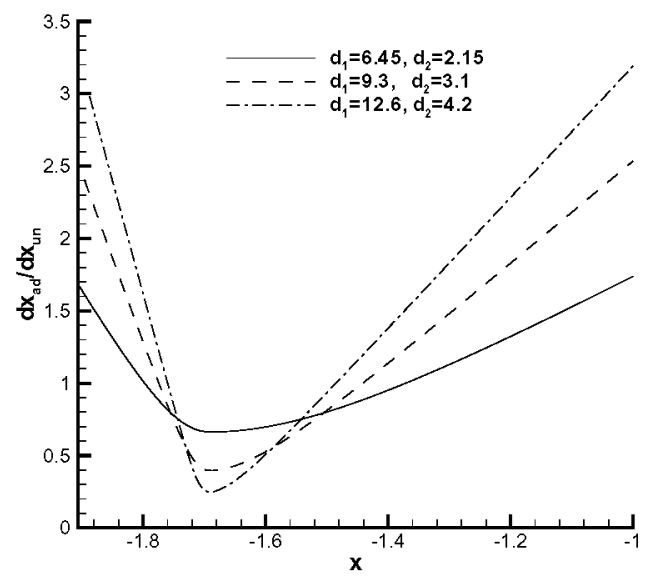

FIG. 3. Ratio of the adaptive grid spacing to the corresponding uniform grid spacing.

is used to generate the adaptive grid:

$$
\begin{aligned}
\tilde{\xi} & =\frac{\int_{r}^{r_{1}} f(r, \theta) d r}{\int_{r_{1}}^{r_{1}} f(\theta)} f(r, \theta) d r \\
\tilde{\eta} & =\theta / \theta_{\max },
\end{aligned}
$$

with the monitor function $f(r, \theta)$ defined as

$$
\begin{aligned}
& f(r, \theta)=\frac{1}{\sqrt{1+\left[d_{3}\left(d_{2}-1\right) \frac{r-r_{s}(\theta)}{r_{s}(\theta)-r 1}\right]^{2}}} \\
& d_{3}=d_{1}+\frac{d_{2}-d_{1}}{1+e^{\delta\left(r-r_{s}(\theta)\right)}},
\end{aligned}
$$

where $r_{s}(\theta)$ is the bow shock radius, $d_{1}, d_{2}$, and $\delta$ are parameters. Note that the same monitor function Eq. (13) is used to generate all adaptive grids, independently of the number of grid points. Such a choice of the monitor function provides that the metric coefficients are $C^{\infty}$ functions in the entire physical domain, which is important for constructing high-order accurate finite difference approximations on nonuniform grids. The ratios of the adaptive grid spacing to the uniform one corresponding to three sets of the parameters $d_{1}$ and $d_{2}\left(d_{1}^{(1)}=6.45\right.$ and $d_{2}^{(1)}=2.15$, $d_{1}^{(2)}=9.3$ and $d_{2}^{(2)}=3.1, d_{1}^{(3)}=12.6$ and $\left.d_{2}^{(3)}=4.2\right)$ used in the numerical calculations are shown in Fig. 3. The figure shows that the adaptive grid spacing achieves its minimum value at the shock, while away from the shock, it behaves as a linear function. This linear dependence, which corresponds to the well-known exponential stretching, provides that in smooth flow regions, the leading truncation error terms resulting from the grid nonuniformity and the approximation of $\hat{\mathbf{F}}_{\xi}$ are of the same order. 


\subsection{Local Grid Refinement Method}

$\Lambda$ s shown above, for the blunt body problem in question, the radial error component is much larger than the circumferential one. Therefore, the local grid refinement method is also employed in a 1-D manner. The resulting computational grid is obtained as a sequence of structured quadrilateral nested grids that are aligned with the shock and arranged in block structures, such that each of them has the same family of radial lines, $\eta=$ const. This local grid refinement procedure en sures that there are no "hanging" nodes on the embedded interfaces and that the smoothness of the original background grid is maintained. Taking into account that the maximum pointwise error occurs at the shock and that the exact shock position is known a priori, a family of the nested grids is generated only in the vicinity of the exact shock location, while the original uniform grid is used in the rest of the domain. The width of the region where the grid is locally refined is an adjustable parameter that is chosen to be larger than a stencil of the numerical scheme used.

This local grid refinement procedure is consistent with the error equidistribution principle which says that the grid point distribution is asymptotically optimal if some error measure is equally distributed over the field. However, for problems with shocks, the error cannot be equidistributed, because the error function is singular at the discontinuity. In this case, the stopping criterion for the local grid refinement is not trivial. In the present analysis, the adaptive mesh refinement method is used to recover the design accuracy of a high-order shock-capturing scheme downstream of the shock. This condition can be used as the stopping criterion, which determines how many levels of refinement are required to guarantee that the numerical solution error obtained on the adaptive grid is consistent with the design order of the scheme employed. Using the heuristic model expressing the first-order nature of any highorder shock-capturing scheme [2], one can represent the total error in the numerical solution as follows:

$$
\epsilon=C_{1} h+C_{p} h^{p}
$$

where $h$ is a grid spacing, $\epsilon$ is the solution error, $p$ is the design order of the numerical algorithm, and $C_{1}$ and $C_{p}$ are problem dependent constants. If the solution smoothness is consistent with the order of the approximation used, then $C_{1}=0$ and $C_{p}$ is proportional to the $(p+1)$ th derivative of the solution. On the other hand, if there are unresolved features in the flow such as shock waves and contact discontinuities, the first-order error component is generated by the shockcapturing procedure, so that $C_{1} \neq 0$. Note that the shock error component may in general include not only the first-order term, but also higher order terms in its expansion. Asymptotically, the shock error component is dominated by the firstorder term $C_{1} h$, whereas the design-order error component is dominated by the leading truncation error term $C_{p} h^{p}$. Therefore, only the leading shock error and design-order error terms are retained in Eq. (14).

$\Lambda$ s follows from Eq. (14), to obtain the $p$ th order accurate solution, the local grid spacing near the shock must be of the order of $H^{p}$, where $H$ is a grid spacing of the background mesh. Despite the simplicity of this criterion, the total number of grid points, and consequently the complexity of the algorithm, can increase significantly. Actually, if a $p$ th-order scheme is used to discretize the governing equations on the 
background uniform mesh with a grid spacing $H$, the local grid size in the vicinity of the shock should be

$$
h=O\left(H^{p}\right)
$$

to obtain a convergence rate of $p$.

$\Lambda$ ssuming that the local grid refinement procedure starts from the background mesh and a nested grid at the next level is refined in the radial direction by a factor of 2, the total number of levels of refinement needed to satisfy Eq. (15) is

$$
l=-(p-1) \log _{2} H
$$

Upon completion of this local grid refinement procedure, the total number of grid points becomes

$$
N_{\text {total }}=1+\frac{1}{H}+2 l N_{\mathrm{w}}
$$

where $N_{\mathrm{w}}$ is a half-width of a region in which the background mesh is locally refined. For example, if one uses a fourth-order scheme on a $65 \times 65$ background mesh, the locally refined grid satisfying condition (15) and corresponding to $N_{\mathrm{v}}=8$ has 353 grid points in the radial direction. Thus, the total number of grid points is increased by a factor of 6 . In practical applications, a grid is refined in both $\xi$ and $\eta$; therefore, the same increase in the number of grid points should be in each spatial direction. Note that the time step $\Delta t$ is also refined by the same factor so that ratios $\Delta t / \Delta \xi$ and $\Delta t / \Delta \eta$ are the same on all nested grids, ensuring the stability with explicit finite difference schemes. $\Lambda$ s follows from the above example, the local grid spacing near the shock would be of the order of $O\left(2^{-24}\right)$, which makes the grid refinement study practically impossible. Therefore, instead of satisfying Eq. (15), we perform three series of calculations on locally refined grids that have a different number of levels of refinement. During the grid refinement study, the background mesh is refined globally. From Eq. (15) it follows that the number of levels of refinement is increased by one. For instance, if a locally refined grid corresponding to a $33 \times 33$ background mesh has three levels of refinement, then on the next $65 \times 65$ background grid, one level of refinement is added, so that the total number of levels becomes four.

Remark 1. The grid adaptation procedures described above are based on the fact that the exact shock location is known a priori. However, in practice, the exact shock position is unknown, which may introduce an additional error in the numerical solution. As has been shown in [16], traditional grid adaptation methods can provide that shocks are well resolved, but their locations are highly inaccurate due to the lack of resolution of smooth portions of the solution.

\section{ANALYSIS OF THE ERROR CAUSED BY GRID NONUNIFORMITY}

5.1. Error Introduced by Grid Redistribution

Let us estimate the error in the smooth portion of the numerical solution, which is introduced by concentrating grid points near the shock. Because grid points are 
redistributed in a 1-D manner in the present study, we consider the following 1-D scalar equation:

$$
\psi_{t}+f_{x}(\psi)=0
$$

which can be treated as a 1-D analog of the hyperbolic portion of the 2-D steady Euler equations (1). It can be shown by rewriting the Euler equations (1) in nonconservative form

$$
\frac{\partial \mathbf{U}}{\partial x}+\mathbf{A} \frac{\partial \mathbf{U}}{\partial y}=0
$$

where $\mathbf{A}=\left(\frac{\partial \mathbf{F}}{\partial \mathbf{U}}\right)^{-1}\left(\frac{\partial \mathbf{G}}{\partial \mathbf{U}}\right)$. The type of the equations (19) is governed by the eigenvalues of the $\mathbf{A}$ matrix, which are

$$
\begin{aligned}
& \lambda_{1,2}=\frac{v}{u} \\
& \lambda_{3,4}=\frac{u v \pm c \sqrt{u^{2}+v^{2}-c^{2}}}{u^{2}-c^{2}},
\end{aligned}
$$

where $c$ is the speed of sound. For the blunt body problem under consideration, eigenvalues $\lambda_{1}$ and $\lambda_{2}$ are always real, regardless of the local Mach number. Therefore, the first-order error generated by the shock-capturing procedure at the shock propagates downstream along the streamlines $v / u$.

$\Lambda$ ssuming that the initial profile $\psi(0, x)$ is discontinuous, the solution error of Eq. (18) downstream of the captured shock consists of the first-order error component caused by the shock capturing and the design-order truncation error component. Thus,

$$
\epsilon=C_{1} \Delta x+T_{p}(x)
$$

where $C_{1}$ is a constant dependent on the solution, $\Delta x$ is a local grid spacing, and $T_{p}$ is the leading truncation error term of a pth-order shock-capturing scheme used for approximation of Eq. (18). Since our aim is to estimate the influence of the spatial nonuniformity on the numerical solution accuracy, the temporal error component is not considered. To the authors' knowledge, very little work has been done to estimate the coefficient $C_{1}$. In the present analysis, it is assumed that the coefficient $C_{1}$ approaches a constant on sufficiently fine grids. This assumption is corroborated by the numerical calculations presented in Section 6 .

To evaluate the leading truncation error term on a nonuniform grid, a $p+1$ times differentiable one-to-one coordinate transformation,

$$
\begin{aligned}
& t=\tau \\
& x=x(\xi),
\end{aligned}
$$

between the physical and computational spaces is considered. The nonuniform grid in the physical space is generated as images of nodes of a uniform mesh in the computational domain $Q$ :

$$
x_{j}=x\left(\xi_{j}\right), \quad \xi_{j}=\frac{j}{J}, j=\overline{0, J} .
$$


It is assumed that $x(\xi)$ is a $C^{\infty}$ function such that $x_{\xi}>0 \forall \xi \in Q$. Transferring the $x$-derivative in Eq. (18) to the computational space, Eq. (18) is rewritten as

$$
\psi_{\tau}+\frac{f_{\hat{\xi}}(\psi)}{x_{\xi}}=0
$$

$\Lambda$ pproximating $f_{\xi}$ and $x_{\xi}$ by some $p$ th-order finite difference formulas yields

$$
L_{h}^{(p)}\left(f_{x}\right)=\frac{\sum_{n=j-n_{1}}^{j+n_{2}} \alpha_{n} f_{n}}{\sum_{m=j-m_{1}}^{j+m_{2}} \beta_{m} x_{m}},
$$

where $L_{h}$ is a finite difference operator; $n_{1}, n_{2}, \alpha_{n}$ and $m_{1}, m_{2}, \beta_{m}$ depend on particular approximations used for evaluating $f_{\xi}$ and $x_{\xi}$, respectively. It should be stressed that the present analysis is performed in regions where the solution is smooth, so it is assumed that all derivatives needed for the derivation are continuous functions on $\xi \in[0,1]$. Expanding the numerator and denominator of Eq. (24) in a Taylor series about $\xi_{j}$ and omitting the index $j$ on the right hand side, we have

$$
\begin{gathered}
\sum_{\substack{n=j-n_{1} \\
j+m_{2}}}^{j+n_{2}} \alpha_{n} f_{n}=f_{\xi}+C_{p}^{f} f_{\xi}^{(p+1)} \Delta \xi^{p}+O\left(\Delta \xi^{p+1}\right) \\
\sum_{m=j-m_{1}} \beta_{m} x_{m}=x_{\xi}+C_{p}^{x} x_{\xi}^{(p+1)} \Delta \xi^{p}+O\left(\Delta \xi^{p+1}\right),
\end{gathered}
$$

where $x_{\xi}^{(p+1)}=\frac{\partial^{p+1} x}{\partial \xi^{p+1}}, f_{\xi}^{(p+1)}=\frac{\partial^{p+1} f}{\partial \xi^{p+1}}, \Delta \xi=1 / J$, and $C_{p}^{f}$ and $C_{p}^{x}$ are constants dependent on $\alpha_{n}$ and $\beta_{m}$, respectively. Substituting Eq. (25) into Eq. (24) and taking into account that $x_{\xi}>0, \forall \xi \in[0,1]$, one can write

$$
L_{h}\left(f_{x}\right)=\frac{f_{\xi}+C_{p}^{f} \Delta \xi^{p} f_{\xi}^{(p+1)}}{x_{\xi}\left(1+C_{p}^{x} \frac{\Delta \xi^{p}}{x_{\xi}} x_{\xi}^{(p+1)}\right)}+O\left(\Delta \xi^{p+1}\right)
$$

If $\Delta \xi$ is chosen to be sufficiently small so that $\Delta \xi^{p}\left|x_{\xi}^{(p+1)} / x_{\xi}\right| \ll 1$, Eq. (26) can be linearized as follows:

$$
L_{h}\left(f_{x}\right)=\frac{1}{x_{\xi}}\left(f_{\xi}+C_{p}^{f} \Delta \xi^{p} f_{\xi}^{(p+1)}\right)\left(1-C_{p}^{x} \frac{\Delta \xi^{p}}{x_{\xi}} x_{\xi}^{(p+1)}\right)+O\left(\Delta \xi^{p+1}\right) .
$$

Note that the error introduced by the linearization is of the order of $O\left(\Delta \xi^{2 p}\right)$. Neglecting higher order terms in Eq. (27), the leading truncation error term can be recast as

$$
T_{p}(x)=L_{h}\left(f_{x}\right)-f_{x}=C_{p}^{f} \Delta \xi^{p} \frac{f_{\xi}^{(p+1)}}{x_{\xi}}-C_{p}^{x} \Delta \xi^{p} \frac{x_{\xi}^{(p+1)}}{x_{\xi}^{2}} f_{\xi} .
$$

With the first-order error component, the solution error downstream of the shock becomes

$$
\epsilon=C_{1} x_{\xi} \Delta \xi+C_{p}^{f} \Delta \xi^{p} \frac{f_{\xi}^{(p+1)}}{x_{\xi}}-C_{p}^{x} \Delta \xi^{p} \frac{x_{\xi}^{(p+1)}}{x_{\xi}^{2}} f_{\xi}
$$


From the above equation it follows that the error in the smooth portion of the solution consists of three parts. The first one is due to the shock-capturing procedure. The second one, which also exists on uniform meshes, arises from the evaluation of $f_{\xi}$. The third one is caused by the grid nonuniformity. When the grid is clustered in the vicinity of the discontinuity, the metric coefficient $x_{\xi}$ changes rapidly, achieving its minimum value at the shock. $\Lambda$ s has been shown earlier, any grid adaptation technique based on the error minimization or the error equidistribution principle concentrates grid points near discontinuities of the solution, because the true error and its estimates reach their maximum values at the singularity. From Eq. (29) it follows that this kind of grid adaptation reduces the first-order error component, but at the same time, the second and, especially, the last term of the

numerical solution error increase drastically in regions where the $x_{\xi}^{(p+1)}$ becomes very large because of the strong grid nonuniformity. In other words, traditional grid adaptation methods based on the grid redistribution technique transfer the error from the first-order term to the design-order term. Although, asymptotically, the solution error is dominated by the first-order shock error, numerical calculations show that the coefficient $C_{1}$ in Eq. (29) may be very small. $\Lambda$ s will be shown in Section 6 , when the blunt body problem is solved by using the second-order fully upwind scheme based on the Lax-Friedrichs flux splitting, the numerical solution error downstream of the shock is dominated by the design-order error component for all the grids considered, i.e., $\left\|C_{1} h\right\|<\left\|C_{p} h^{p}\right\|$. $\Lambda$ s a result, the first-order error reduction owing to the grid adaptation is much less than the high-order error introduced by clustering grid points near the shock, which ultimately decreases the overall solution accuracy.

\subsection{Error Introduced by Local Grid Refinement}

In contrast to the grid redistribution method, the local grid refinement approach described earlier does not change the metric tensor of the mapping and, therefore, the error component resulting from the high-order derivatives of the metric coefficients remains the same as on the nonadaptive background mesh. Despite this advantage, the local grid refinement introduces additional error caused by an abrupt change in grid spacing at interfaces between coarse and fine grids and by interface boundary conditions.

To illustrate the main problems associated with the local grid refinement method, we investigate wave propagation properties when there is a discontinuity in grid spacing. $\Lambda$ scalar linear equation,

$$
\Phi_{x}+a \Phi_{y}=0,
$$

is considered as a model problem, where $\boldsymbol{a}$ is a positive constant representing tangent of the angle between the freestream flow and the $x$-axis. In the numerical calculations, the parameter $a$ was chosen to be 0.34 . The model equation which can be treated as a scalar analog of the Euler equations (19) is solved on a unit square with the following boundary conditions: $\Phi(0, y)=\mathrm{e}^{I \omega y}$ and $\Phi(x, 0)=\mathrm{e}^{-I \omega a x}$. To simplify the analysis, we seek the solution of Eq. (30) in the following form:

$$
\Phi(x, y)=\varphi(x) \mathrm{e}^{I \omega y}
$$


where $I=\sqrt{-1}$. Substituting Eq. (31) in Eq. (30) yields

$$
\varphi_{x}+\operatorname{Ia\omega } \varphi=0
$$

The above equation is approximated by using a second-order fully upwind scheme in the interior and a two-point first-order upwind approximation at the inflow boundary. First, we consider a uniform grid with grid spacing $h$. In this case, the corresponding discrete equations are

$$
\frac{3}{2} \varphi_{j}-2 \varphi_{j-1}+\frac{1}{2} \varphi_{j-2}+\operatorname{Ia} h h \varphi_{j}=0, \quad j=\overline{2, J}
$$

and

$$
\varphi_{1}-\varphi_{0}+\operatorname{Ia\omega h} \varphi_{1}=0
$$

Introducing a parameter $\alpha=a w h$, equation (33) becomes

$$
\left(\frac{3}{2}+\alpha I\right) \varphi_{j}-2 \varphi_{j-1}+\frac{1}{2} \varphi_{j-2}=0
$$

Substituting the solution of the form

$$
\varphi_{j}=c \lambda^{j}
$$

into the discrete equation (35) yields

$$
\left(\frac{3}{2}+\alpha I\right) \lambda^{2}-2 \lambda+\frac{1}{2}=0 .
$$

The quadratic equation can easily be solved to give

$$
\lambda_{1,2}=\frac{1 \pm \sqrt{1-\frac{1}{2}\left(\frac{3}{2}+\alpha I\right)}}{\frac{3}{2}+\alpha I} .
$$

Thus, the general solution of Eq. (35) is given by

$$
\varphi_{j}=c_{1} \lambda_{1}^{j}+c_{2} \lambda_{2}^{j}
$$

The unknown coefficients $c_{1}$ and $c_{2}$ are found by using the initial condition $\left.\varphi\right|_{x=0}=$ $\varphi_{0}$ and $\mathbf{E q} .(34)$, as follows:

$$
\begin{aligned}
& c_{1}+c_{2}=\varphi_{0} \\
& (1+\alpha I)\left(c_{1} \lambda_{1}+c_{2} \lambda_{2}\right)-c_{1}-c_{2}=0 .
\end{aligned}
$$

The exact discrete solution Eqs. $(31,38-40)$ has been derived under the assumption that the computational grid is uniform. However, the same approach can be applied to construct the discrete solution on a locally refined grid. Without loss of generality, only a two-level locally refined grid with a factor of two refinement, i.e., $h_{1} / h_{2}=2$, is considered. The exact discrete solution on the locally refined grid can 
be constructed as follows. $\Lambda$ ssuming that the coarse grid with grid spacing $h_{1}=h$ covers the left half of the domain, and that the discontinuity in mesh size is located at the midpoint $x=1 / 2$, the solution at this point is

$$
\varphi_{J / 2}^{(1)}=c_{11} \lambda_{11}^{J / 2}+c_{12} \lambda_{12}^{J / 2}
$$

where $c_{11}=c_{1}, c_{12}=c_{2}$, and $\lambda_{11}$ and $\lambda_{12}$ are roots of the characteristic polynomial Eq. (37) corresponding to the background mesh $h_{1}=h$. The above numerical solution can be treated as an incident wave traveling from left to right, which is then transmitted by the interface between the coarse and fine grids. For the hyperbolic equation (30), the solution of the Riemann problem at the interface is trivial $\varphi_{\text {right }}=\varphi_{\text {left }}$. Since the same fully upwind scheme Eq. (33) is used in the right half of the domain, the discrete solution on the fine nested grid can be written in the same form as Eq. (38-40). However, instead of the grid spacing $h_{1}=h$, one should use the grid spacing $h_{2}=h / 2$, and the Direchlet boundary condition at $x=0$ should be replaced with the solution of the Riemann problem at the midpoint, i.e., $\left.u\right|_{x=1 / 2}=\varphi_{J / 2}$, where $\varphi_{J / 2}$ is given by Eq. (41). Thus, the exact discrete solution on the locally refined mesh is

$$
\varphi_{j}^{(2)}=c_{21} \lambda_{21}^{j}+c_{22} \lambda_{22}^{j}
$$

where $\lambda_{21}$ and $\lambda_{22}$ are defined by Eq. (38) with $\alpha=a w h_{2}$, and the coefficients $c_{21}$ and $c_{22}$ are the solution of the following linear system of equations:

$$
\begin{aligned}
& c_{21}+c_{22}=\varphi_{J / 2}^{(1)} \\
& (1+\alpha I)\left(c_{21} \lambda_{21}+c_{22} \lambda_{22}\right)-c_{21}-c_{22}=0 .
\end{aligned}
$$

The exact discrete solution Eqs. (42-43) calculated on the locally refined grid is compared with the uniform grid solution at $x=1$. To determine the influence of the grid discontinuity on the numerical solution accuracy, we compare the error obtained at $x=1$ on both the locally refined and corresponding uniform grids. The error is calculated as the absolute value of the difference between the discrete and analytical solutions of the original differential equation (30), which is

$$
\Phi(x, y)=\mathrm{e}^{-\operatorname{Ia\omega x}} \mathrm{e}^{I \omega y}
$$

The ratio of the error obtained on the locally refined mesh to the uniform grid solution error corresponding to four grid spacings of the background mesh $\left(h=10^{-2}, 10^{-3}, 10^{-4}\right.$, and $\left.10^{-5}\right)$ is plotted versus $\omega^{3} h^{2}$ in Fig. 4. Note that the parameter $\omega^{3} h^{2}$ has been chosen so that it is proportional to the leading truncation error term of the second-order scheme used. Therefore, for small values of $\omega^{3} h^{2}$ corresponding to the fully resolved solution, the error ratios calculated on grids with different background grid spacings are practically identical. The curves corresponding to different mesh densities begin to diverge at a critical value of $\omega^{3} h^{2}$ that corresponds to the point where the error obtained on the locally refined mesh becomes equal to the error obtained on the background mesh. Since this point is asymptotically independent of the grid spacing, we can introduce the critical 


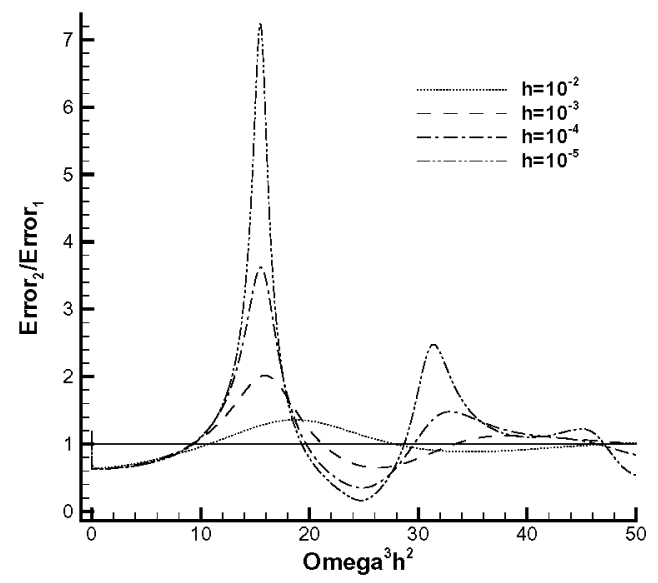

FIG. 4. Ratio of the error obtained on the two-level locally refined grid to the error obtained on the corresponding background grid.

frequency

$$
\omega_{*} \approx\left(\frac{9.3}{h^{2}}\right)^{1 / 3}
$$

If $0<\delta<\omega<\omega_{*}$, where $\delta$ is a small positive constant, then the locally refined grid provides better accuracy than the corresponding background mesh. Note that the constant in the formula (45) for the critical frequency $\omega_{*}$ depends on the numerical scheme used, the angle between the freestream flow and the $x$-axis, and the size of the domain. Despite this dependence, for any stable second-order scheme, the qualitative behavior $\omega_{*} \sim h^{-2 / 3}$ always remains the same.

If the frequency of the exact solution exceeds the critical value $\omega_{*}$, it cannot be resolved on a uniform grid with the grid spacing $h$. $\Lambda$ s a result, the errors ratio exhibits oscillatory behavior. Note, however, that for all the grids considered, there exists the local maximum at $\omega^{3} h^{2} \approx 15$, corresponding to the frequency that is most strongly amplified by the abrupt change in grid spacing, as is evident in Fig. 4. The main reason for such a behavior is the hyperbolic nature of Eq. (30). Actually, if error has been introduced on the coarse grid, it propagates downstream along the characteristic. In the case of discontinuous solutions, such as shock waves, the captured discontinuity involves all frequencies and, therefore, cannot be fully resolved on any grid. The error component corresponding to these unresolved frequencies propagates downstream along the characteristics and amplifies at interfaces. The error amplification occurs because of the discontinuity in grid spacing and the approximate interface boundary conditions. It should be emphasized that the error amplification becomes stronger, if either the number of interfaces or the grid refinement factor increases, or the grid is globally refined.

$\Lambda$ s one can see in Fig. 4, there is a boundary layer at $\omega^{3} h^{2} \rightarrow 0$. This deterioration in accuracy for the lowest frequencies is caused by reducing by one the order of approximation at the first point of the coarse and fine grids. To demonstrate this 
property; we estimate the ratio of the solution error obtained on the locally refined mesh to one obtained on the corresponding uniform grid, when the exact solution frequency is sufficiently small, i.e.,

$$
\omega \ll h^{-2 / 3} .
$$

If inequality (46) holds, then the truncation error analysis can be applied. Taking into account the fact that the error of integration of Eq. (32) is proportional to the length of the integration interval, the ratio of the truncation errors can be estimated as follows:

$$
\frac{\epsilon_{2}}{\epsilon_{1}}=\left|\frac{\left[C_{1} \varphi_{x x} h^{2}+C_{2} \varphi_{x x x} h^{2}\left(\frac{1}{2}-h\right)\right]+\left[C_{1} \varphi_{x x}\left(\frac{h}{2}\right)^{2}+C_{2} \varphi_{x x x}\left(\frac{h}{2}\right)^{2}\left(\frac{1}{2}-\frac{h}{2}\right)\right]}{C_{1} \varphi_{x x} h^{2}+C_{2} \varphi_{x x x} h^{2}(1-h)}\right|,
$$

where $C_{1}$ and $C_{2}$ are constants dependent on the first- and second-order approximations used, e.g., in our case $C_{1}=-1 / 2$ and $C_{2}=-1 / 3$. In Eq. (47), the first term in the square brackets is the integration error on the coarse grid with grid spacing $h$, and the second term is the integration error obtained on the fine grid with grid spacing $h / 2$. Note that the $C_{1} \varphi_{x x} h^{2}$ and $C_{1} \varphi_{x x}(h / 2)^{2}$ terms are due to the first-order approximation used at the first grid points of the coarse and fine grids, respectively. With the exact solution Eq. (44), Eq. (47) is reduced to

$$
\frac{\epsilon_{2}}{\epsilon_{1}}=\left|\frac{\frac{5}{4} C_{1}+I C_{2} \omega\left(\frac{1}{2}-h\right)+I C_{2} \frac{\omega}{4}\left(\frac{1}{2}-\frac{h}{2}\right)}{C_{1}+I C_{2} \omega(1-h)}\right| .
$$

Letting $h \rightarrow 0$ yields

$$
\frac{\epsilon_{2}}{\epsilon_{1}}=\frac{5}{4\left(C_{1}^{2}+C_{2}^{2} \omega^{2}\right)} \sqrt{C_{1}^{4}+\frac{1}{4} C_{2}^{4} \omega^{4}+\frac{5}{4} C_{1}^{2} C_{2}^{2} \omega^{2}} .
$$

From the above equation it follows that for the lowest frequency $\omega \rightarrow 0$, the truncation error calculated on the globally uniform grid is $5 / 4$ times less than that obtained on the locally refined grid having one and a half times as many grid cells as the corresponding uniform mesh. $\Lambda$ s the wave frequency increases, the errors ratio tends to its asymptotic value of $5 / 8$. Note that if the governing equation is approximated with a second-order scheme in the entire computational domain, then the first-order terms in Eq. (47) vanish, providing that $\epsilon_{2} / \epsilon_{1}=5 / 8, \forall \omega: \omega \ll h^{-2 / 3}$.

Remark 2. In solving the 2-D Euler equations, two additional sources of errors, which are not present in the test example considered above, can arise. The first one is due to an approximate solution of the Riemann problem at interfaces between the coarse and fine grids. The second source of error is caused by the reflection of waves going through interfaces. Note that in the above example, there is no numerical reflection, because the fully upwind formulation, which is consistent with the characteristic of Eq. (30), is employed.

\section{RESULTS AND DISCUSSION}

The inviscid $M_{\infty}=3$ flow around a circular cylinder is used to test the accuracy of the grid redistribution and local grid refinement methods. $\Lambda$ grid refinement study 
is performed to investigate the influence of the grid adaptation on the numerical solution accuracy downstream of the captured bow shock. The following sequence of grids is used in the grid refinement study: $33 \times 33,65 \times 65,129 \times 129$, and $257 \times 257$. Error in the smooth portion of the solution is measured in the $L_{2}$ sense, as follows:

$$
\left\|\phi-\phi^{\mathrm{ex}}\right\|_{L_{2}}=\sqrt{\frac{\sum_{k=1}^{K} \sum_{j=1}^{J}\left(\phi_{k j}-\phi_{k j}^{\mathrm{ex}}\right)^{2}}{K J}},
$$

where $\phi_{k j}$ and $\phi_{k j}^{\mathrm{ex}}$ are the numerical and exact values of $\phi$ at point $(k, j)$, and $K$ and $J$ are the number of grid points in the domain of interest. The norm of wall quantities is formed in a manner similar to Eq. (50), but only the wall points are used in the formula.

\subsection{Grid Redistribution Method}

$\Lambda \mathrm{n}$ adaptive grid is generated by equidistributing the monitor function Eq. (13), as described in Section 4.1. Three sets of the parameters $d_{1}$ and $d_{2}(6.45$ and 2.15, 7.95 and $2.65,12.6$ and 4.2) in Eq. (13) generate three families of the adaptive grids. These three sets of parameters $d_{1}$ and $d_{2}$ provide that the ratio of the adaptive grid spacing to the uniform grid spacing at the shock is equal to $1.5,2.5$, and 4 , respectively. $\Lambda$ sample $65 \times 65$ adaptive grid generated by this grid redistribution procedure is shown in Fig. 5. The region around the shock is well resolved by reducing the local grid spacing by a factor of 2.5 compared with the uniform mesh with the same number of grid points. Figure 6 shows the pressure contours obtained with the LF-2-2 scheme on the $65 \times 65$ uniform (left) and adaptive (right) grids. $\Lambda$ s one might expect, the grid adaptation improves the shock resolution. To give greater insight into how the grid clustering influences on the shock smearing, the centerline pressure distributions computed on these adaptive and uniform grids

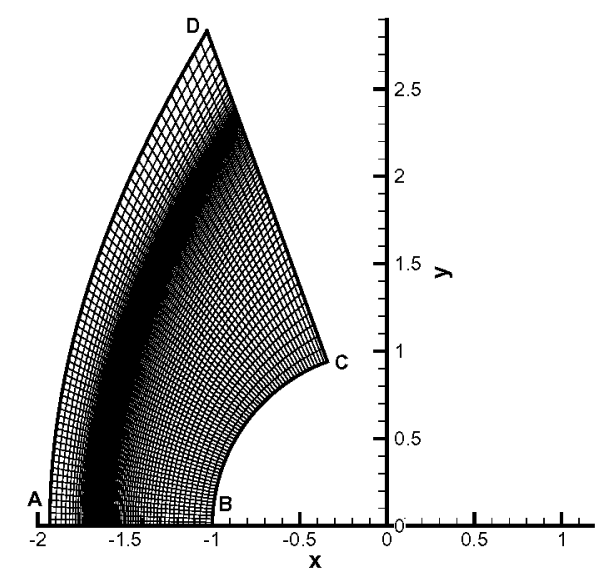

FIG. 5. Adaptive $65 \times 65$ grid $\left(\Delta x_{\mathrm{un}} / \Delta x_{\text {min }}=2.5\right)$ generated by the grid redistribution method. 

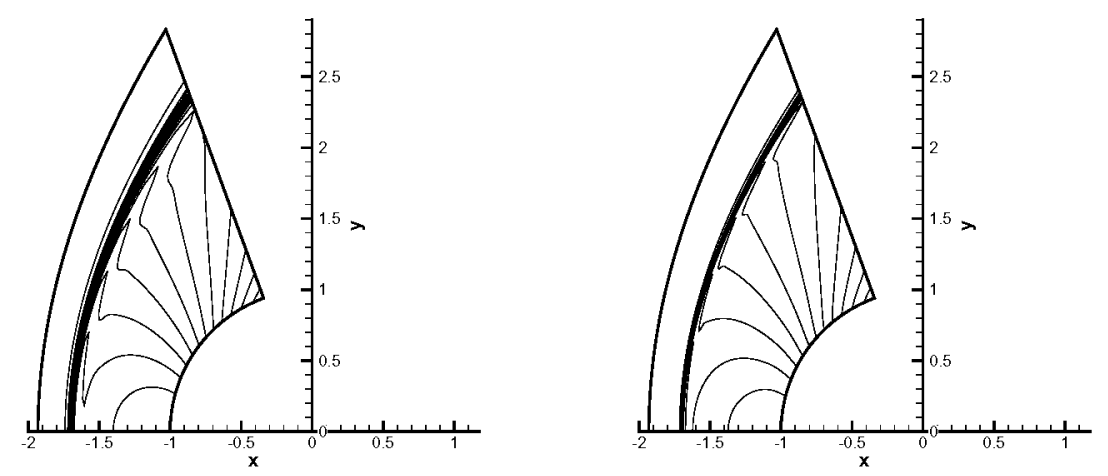

FIG. 6. Pressure contours obtained with the LF-2-2 scheme on $65 \times 65$ uniform (left) and adaptive (right) grids.

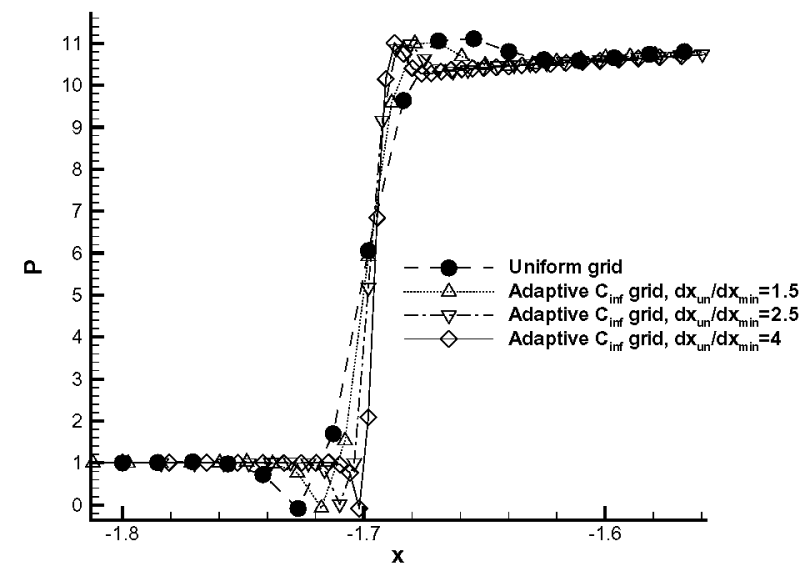

FIG. 7. Centerline pressure distributions calculated using the LF-2-2 scheme on the $65 \times 65$ uniform and adaptive grids.

are depicted in Fig. 7. For all the grids considered, the bow shock is captured within 6 cells, which is typical for the Lax-Friedrichs flux splitting employed. $\Lambda \mathrm{t}$ the same time, the shock thickness, which is proportional to the local grid spacing in the physical space, becomes thinner as the clustering of grid points increases. Locally near the shock, the numerical solution profile, which remains practically unchanged, is scaled according to the local grid spacing. Note that the numerical solutions obtained with the LF-4-3 finite difference scheme demonstrate similar behavior.

Grid refinement studies using three families of adaptive grids and one family of uniform grids that have the same number of grid points are presented in Figs. 8-13. The $L_{2}$ norm of the wall pressure error is shown in Fig. 8. As one can see in the figure, the convergence rate obtained for the smooth problem is consistent with the design order of the scheme used. The smooth problem is formulated in the region 


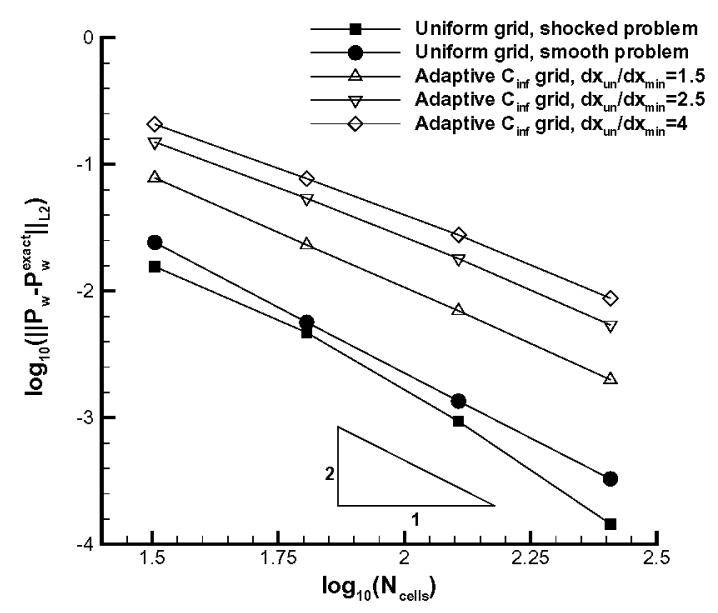

FIG. 8. Grid refinement study at $M_{\infty}=3$, showing the wall pressure error obtained with the LF-2-2 scheme on uniform and adaptive grids.

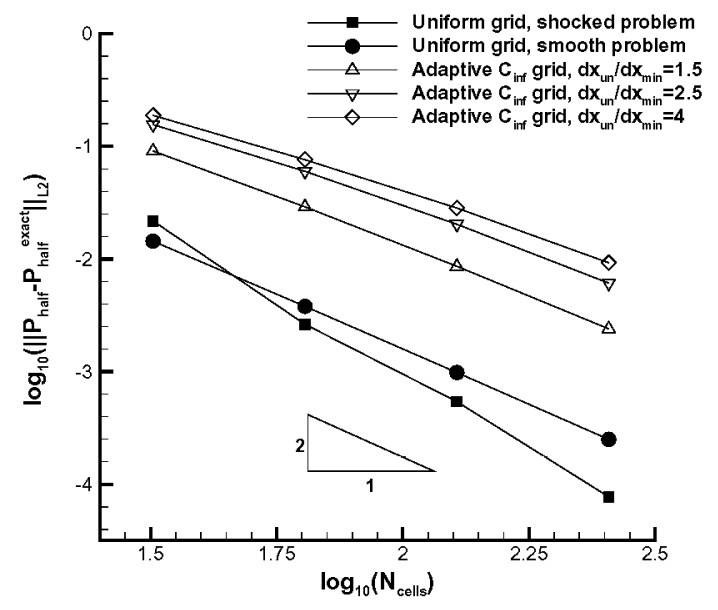

FIG. 9. Grid refinement study at $M_{\infty}=3$, showing the pressure error in the half of the domain closest to the body obtained with the LF-2-2 scheme on uniform and adaptive grids.

bounded by the exact shock wave and the cylinder, so that there is no discontinuity in the domain. The inflow boundary condition at the bow shock is implemented by solving the Riemann problem between the numerical state and the exact post-shock conditions from the spectral solution.

Although the shock resolution is improved by clustering grid points around the shock, the error in the pressure on the body surface increases by a factor of 5-10 compared with that calculated on the uniform grid with the same number of grid points. It should be noted that the higher the concentration of grid points in the vicinity of the shock, the larger the error that is introduced into the numerical solution by the grid nonuniformity. The grid adaptation results in the metric coef- 


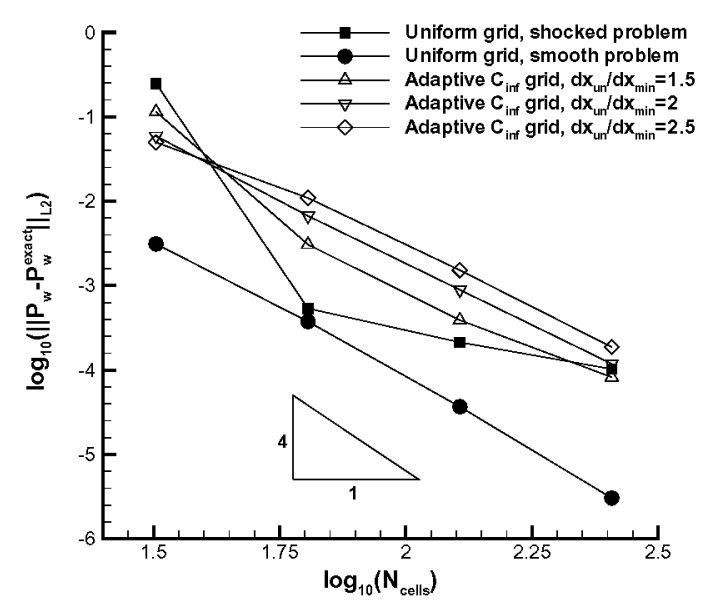

FIG. 10. Grid refinement study at $M_{\infty}=3$, showing the wall pressure error obtained with the LF-4-3 scheme on uniform and adaptive grids.

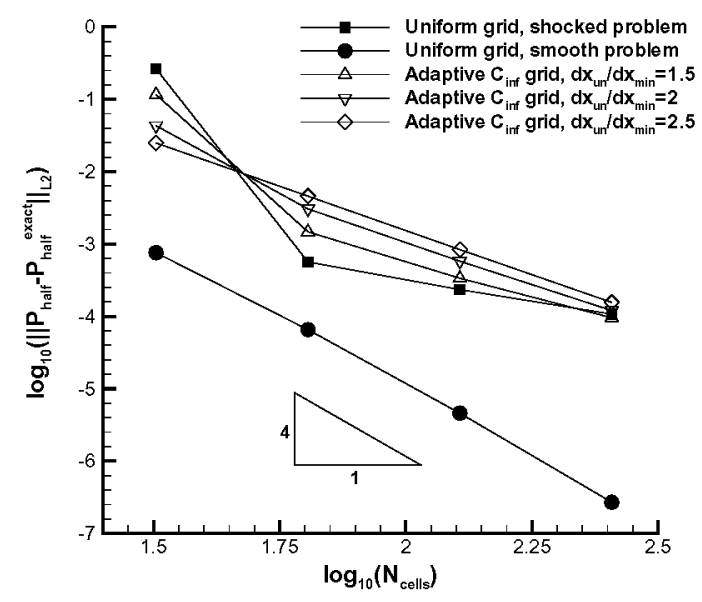

FIG. 11. Grid refinement study at $M_{\infty}=3$, showing the pressure error in the half of the domain closest to the body obtained with the LF-4-3 scheme on uniform and adaptive grids.

ficients drastically increasing near the shock, which, in turn, considerably decreases the accuracy in regions where the solution is smooth. These numerical calculations corroborate the analysis presented in Section 5.1. Surprisingly, the shock-capturing solutions obtained on uniform grids not only are more accurate than the corresponding solution of the smooth problem, but also exhibit higher convergence rate on fine meshes. This anomalous behavior present in the shock aligned cases did not generalize to non-shock aligned meshes. Nevertheless, this superconvergence is not yet well understood and requires additional investigation.

The $L_{2}$ norm of the pressure error calculated in the half of the domain closest to the body is presented in Fig. 9. Such a choice of the domain enables us to exclude 
any point close to the captured shock, where the pointwise error is of the order of $O(1)$. The error behavior obtained in the field is very similar to that exhibited by the wall pressure error. $\Lambda$ s in the foregoing comparison, the grid adaptation significantly increases the error in the smooth portion of the solution. Note that for the LF-2-2 scheme, the first-order error component is comparable with the designorder error, giving the appearance of the second-order convergence rate on all the grids considered.

Error convergence plots obtained with the fourth-order upwind-biased LF-4-3 scheme are depicted in Figs. 10 and 11 which are analogous to Figs. 8 and 9, accordingly. In contrast to the second-order scheme, the fourth-order method using uniform grids quickly approaches the asymptotic limit and exhibits just the firstorder convergence on fine grids. In spite of the fact that the solution error is dominated by the first-order error component, the grid adaptation improves the numerical solution accuracy only asymptotically. On one hand, the concentration of grid points near the shock reduces the first-order error component, so that the convergence rate increases towards the design order limit, as shown in Figs. 10 and 11. On the other hand, the high-order error component significantly increases because of the grid nonuniformity. $\Lambda$ s a result, the solution error is dominated by the design-order error component. Despite some improvement in accuracy on the coarsest adaptive grids, the $L_{2}$ norm of the solution error obtained on finer adaptive grids is larger than that calculated on the corresponding uniform grids. $\Lambda$ s shown in Section 5.1, the main reason for such a behavior is that the designorder error component, resulted from the approximation of the metric coefficients, drastically increases, owing to the clustering of grid points near the shock. Although the adaptive grid solution exhibits a higher convergence rate, the errors obtained on the corresponding adaptive and uniform grids become comparable only on the finest $257 \times 257$ mesh. Hence, the grid redistribution method based on the LF4-3 scheme provides improvement in accuracy only on extremely fine grids which are very seldom found in practical applications, because of the computational cost involved. Note that the adaptive grid solution error suffers from the first-order degeneration but with much lower first-order error constant, as follows from Eq. (29).

In the grid convergence studies presented above, the solution error is measured in regions where the solution is smooth. However, it appears intuitively that the grid adaptation should reduce the solution error in the vicinity of the shock. One can argue that calculation of the lift or the drag for discontinuous flows results in an error in these integral quantities of $O\left(\Delta P_{s} \Delta x_{s}\right)$, where $\Delta P_{s}$ and $\Delta x_{s}$ are the pressure jump across the shock and the grid spacing at the shock, respectively. This assumption is the basis for using grid adaptation methods to reduce the firstorder shock error in the lift and drag which are two of the most important integral quantities obtained from the solution of the Euler equations.

To check whether the grid adaptation improves the accuracy of the integral quantities, we compare errors in the pressure integral across the bow shock along line $y=0$ calculated on the same adaptive and uniform grids. Two integration algorithms have been used to compute the integral. The first one is a standard two-point second-order trapezoidal rule quadrature formula. The second one uses a piecewise cubic spline to fit the discrete pressure. Then, the spline is integrated 


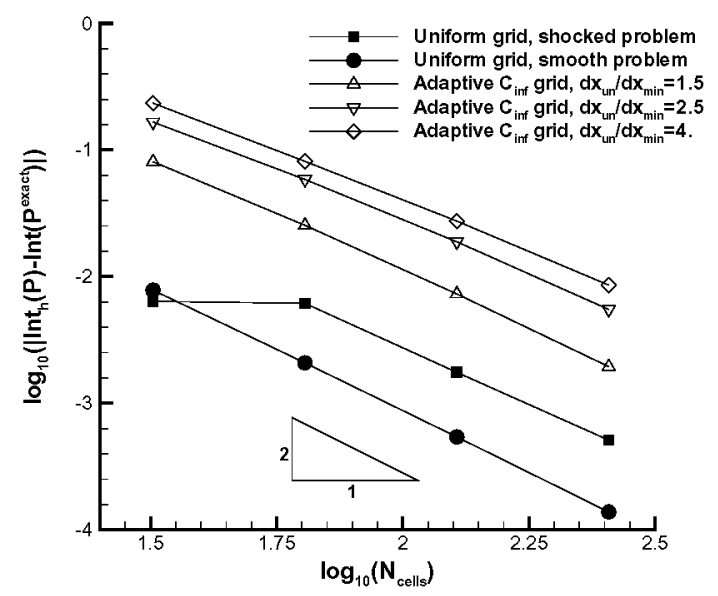

FIG. 12. Grid refinement study for the pressure integral error obtained with the LF-2-2 scheme on uniform and adaptive grids.

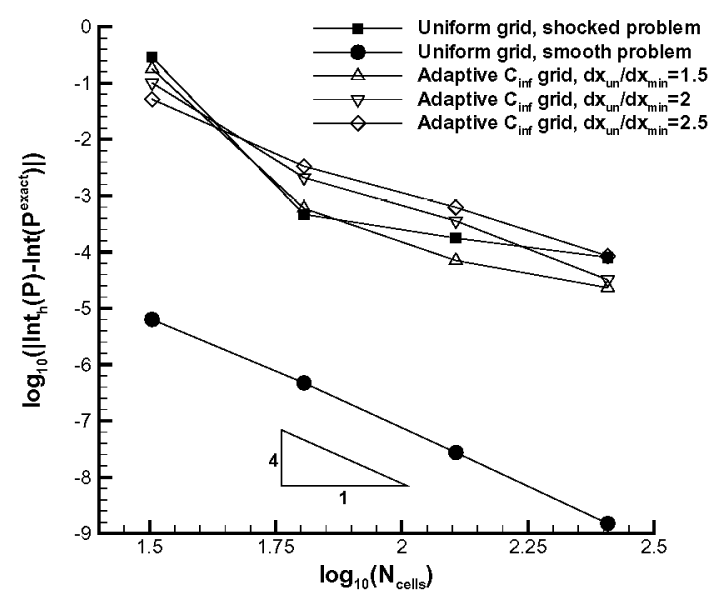

FIG. 13. Grid refinement study for the pressure integral error obtained with the LF-4-3 scheme on uniform and adaptive grids.

analytically, providing a fourth-order accurate integration formula for sufficiently smooth functions. Nlthough the fourth-order integration procedure imposes more severe constraints on smoothness of the integrated function, pressure integral errors obtained with second- and fourth-order quadrature formulas are practically identical. Therefore, only the results calculated using the fourth-order integration procedure are presented.

Figures 12 and 13 show the grid refinement study for the pressure integral obtained with the LF-2-2 and LF-4-3 shock-capturing schemes, respectively. In contrast to conventional wisdom, the pressure integral calculated with the LF-2-2 scheme on clustered grids is less accurate than that computed on uniform grids 
with the same number of grid points. Figure 12 shows that the accuracy of the pressure integral deteriorates as the concentration of grid points in the vicinity of the shock increases. For the LF-4-3 scheme, slight improvement of the pressure integral accuracy can be observed on both the coarsest and finest adaptive grids, while similar deterioration in accuracy occurs on $65 \times 65$ and $129 \times 129$ meshes.

It should be emphasized that the error in the pressure integral across the shock behaves very similarly to the $L_{2}$ norm of the wall pressure error. This is no surprise because these quantities are closely connected. To show this relation, we integrate the 2-D steady Euler equations over the entire domain to give

$$
\int_{V}\left(\mathbf{F}_{x}+\mathbf{G}_{y}\right) d V=\int_{\Omega} \mathcal{F} \cdot \mathbf{n} d \Omega=0
$$

where $\mathcal{F}$ is the flux tensor, $\Omega$ is the boundary of the physical domain $\mathrm{V}, \mathbf{n}$ is an outward pointing unit vector normal to $\Omega$. As follows from Fig. $5, \Omega=A B+B C+$ $C D+D A$. Thus,

$$
\int_{A B} \mathcal{F} \cdot \mathbf{n} d \Omega=-\int_{B C} \mathcal{F} \cdot \mathbf{n} d \Omega-\int_{C D} \mathcal{F} \cdot \mathbf{n} d \Omega-\int_{D A} \mathcal{F} \cdot \mathbf{n} d \Omega .
$$

Because the finite difference schemes used are fully conservative, Eq. (52) holds not only for the exact analytical solution, but also for the discrete solution. $\Lambda$ s follows from Eq. (52), the accuracy of the solution integral along the centerline $A B$ is determined by the accuracy of the integrals calculated along the other boundaries including the body surface $B C$. This indicates that the integral $L_{2}$ norm of the wall pressure error is closely related to the accuracy of the pressure integral along the centerline.

\subsection{Local Grid Refinement Method}

$\Lambda$ s follows from the analysis presented in Section 5.2, the most troublesome parts of the local grid refinement method are the grid discontinuity and the interface boundary conditions. Therefore, special attention has been paid to implementation of the interface boundary conditions and their accuracy. In the present study, the Roe's approximate Riemann solver is used to build a flux at grid interfaces. The left and right states at a grid interface are obtained as solutions of the Euler equations calculated separately in each subdomain. The numerical flux built this way is then used to construct the second- and fourth-order approximations, Eqs. (89). This implementation of the interface boundary conditions maintains stability, conservation, and accuracy in multiple dimensions for both second- and fourthorder methods. Because only matching nested grids are considered, there are no hanging nodes in the domain.

To verify the accuracy of the interface boundary conditions described above, two calculations of the smooth $M_{\infty}=2.5$ blunt-body problem are performed. The first grid refinement study is done on a sequence of uniformly spaced grids. Each grid is divided on eight subdomains containing the same number of grid points in both the radial and circumferential directions, as shown in Fig. 14. Pressure contours computed with the LF-4-3 scheme on this multiblock grid are also shown in Fig. 14. 

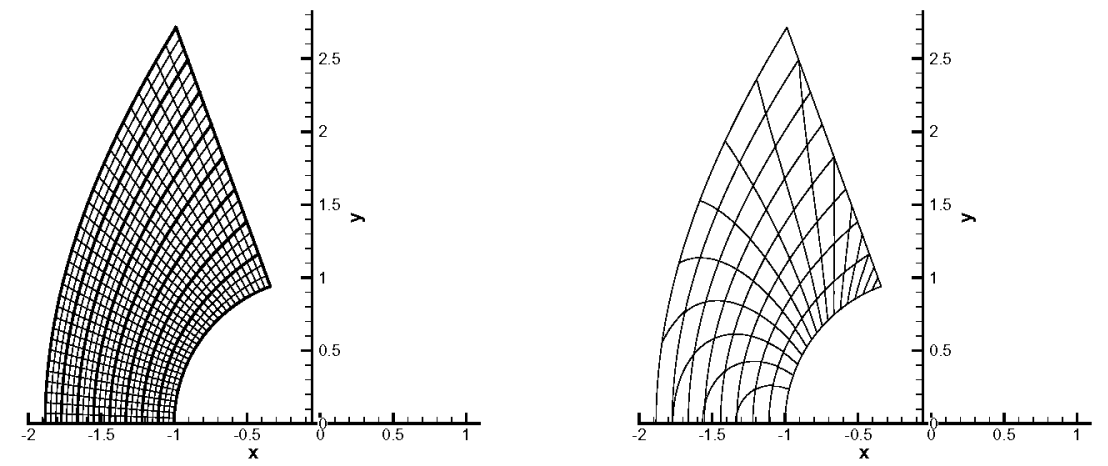

FIG. 14. 8-block $25 \times 33$ uniform grid (left) and pressure contours (right) of the smooth $M_{\infty}=2.5$ flow calculated with the LF-4-3 scheme.
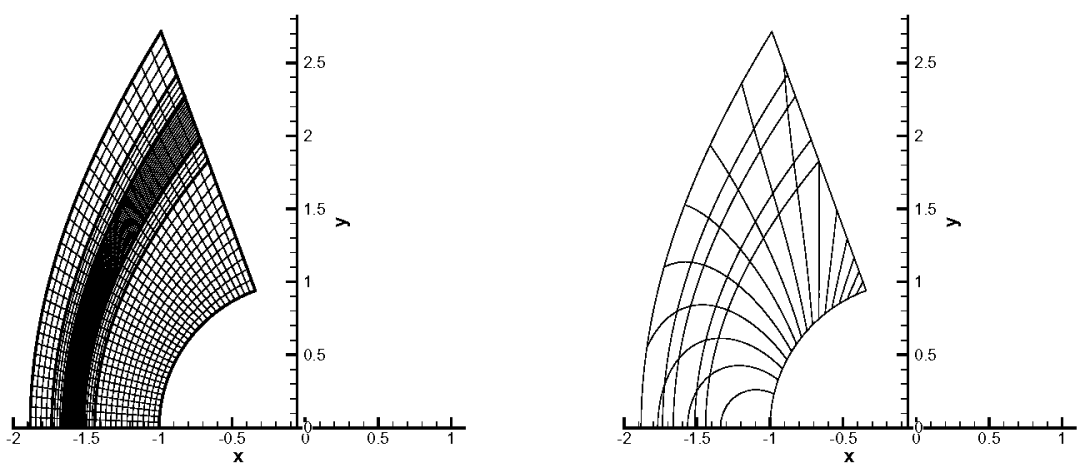

FIG. 15. Two-level $41 \times 33$ locally refined grid (left) and pressure contours (right) of the smooth $M_{\infty}=2.5$ flow calculated with the LF-4-3 scheme.

Nlthough the present formulation of the interface boundary conditions results in the numerical solution being discontinuous at interfaces, the absolute value of the solution jump at the interface is of the order of the truncation error of the scheme used. For example, the characteristic interface pressure jump calculated with the LF-4-3 scheme on a $25 \times 33$ uniform 8-block grid is $O\left(10^{-3}\right)$.

The second series of calculations is performed on a sequence of locally refined grids similar to those which are used in the shock-capturing formulation. $\Lambda$ s in the previous test case, only the flow between the bow shock and the cylinder is considered, i.e., there are no discontinuities in this subproblem. $\Lambda$ three-level locally refined grid corresponding to a $25 \times 33$ background mesh and the pressure contours calculated on this grid are shown in Fig. 15. Qualitatively, the solutions obtained on uniform and nested multiblock grids are very similar. However, the corresponding $L_{2}$ pressure error norms calculated in the half of the domain closest to the body are quite different, as seen in Fig. 16. $\Lambda$ s shown in Section 5.2, the presence of interfaces in the domain introduces additional error in the numerical solution. On the coarsest 8-block uniform grid, the solution is less accurate than that obtained on 


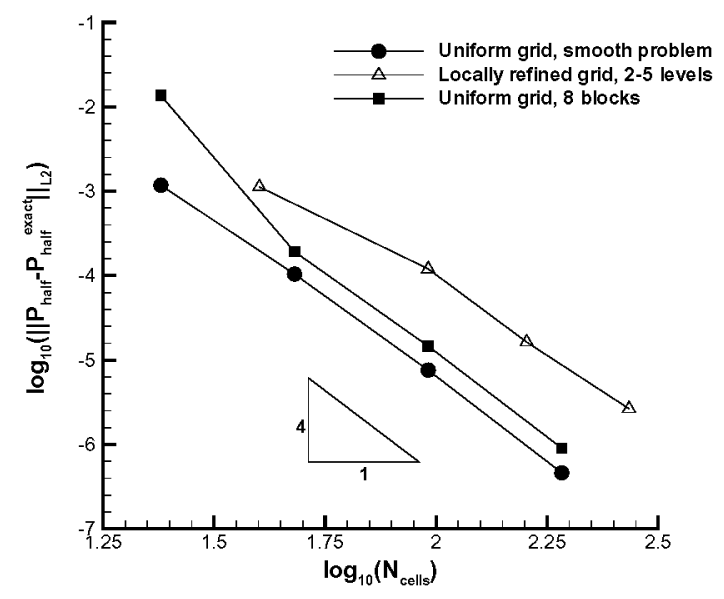

FIG. 16. Grid refinement study at $M_{\infty}=2.5$, showing the pressure error in the half of the domain closest to the body obtained with the LF-4-3 scheme on the 8-block uniform and 2to 5-level locally refined grids.

the corresponding single-block uniform grid. This reduction in accuracy is due to the fact that all grid points in each subdomain are treated as the boundary points. Therefore, the third-order boundary closure approximation is used on the entire coarsest mesh. On finer meshes, the multiblock uniform grid formulation exhibits the design-order convergence rate and provides practically the same accuracy as on the single-block uniform grid. In contrast to multiblock uniform grid results, the locally refined grid formulation leads to both one order of magnitude reduction in accuracy and deterioration of the convergence rate to 3 on fine meshes. These numerical results corroborate the theoretical analysis which shows that the error components corresponding to the lowest fully resolved and the high unresolved frequencies are amplified by the grid discontinuity. Such a reduction in accuracy is also caused by the approximate solution of the Riemann problem and by spurious reflection of waves traveling through the interfaces.

To test the accuracy of the local grid refinement method, the LF-2-2 and LF-4-3 schemes are used to capture the bow shock around a Mach 3 circular cylinder. $\Lambda$ typical three-level $137 \times 65$ locally refined grid used in both second- and fourthorder formulations is shown in Fig. 17. This adaptive grid corresponding to $65 \times 65$ background uniform mesh is locally refined near the exact shock location, such that the local grid spacing of the finest nested subgrid is 8 times smaller than that of the background mesh. Figure 18 shows the pressure contours calculated on these uniform and locally refined grids. $\Lambda$ s expected, the shock wave front becomes much sharper on the adaptive grid. To demonstrate the improvement in the shock resolution owing to the local grid refinement, the centerline pressure distributions computed on the three-level and five-level locally refined grids are compared with the uniform grid results in Fig. 19. The comparison shows that the bow shock is smeared over six grid cells for all the grids considered, while the shock thickness in the physical space is reduced proportionally to the local grid spacing. 


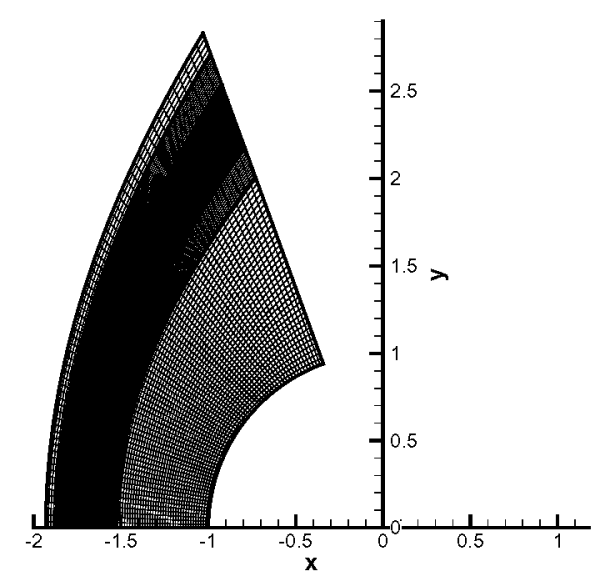

FIG. 17. Three-level $137 \times 65$ locally refined grid.
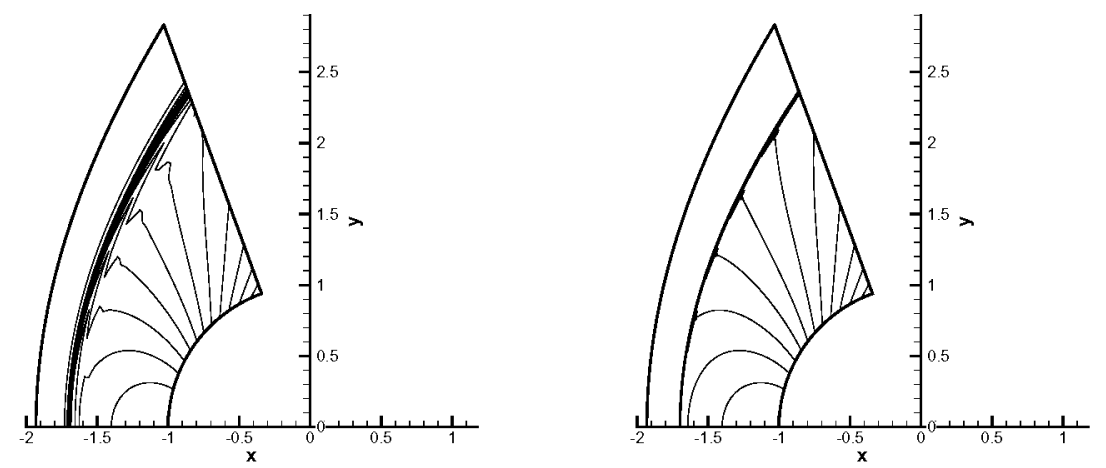

FIG. 18. Pressure contours obtained with the LF-4-3 scheme on the $65 \times 65$ uniform (left) and 3-level $137 \times 65$ locally refined (right) grids.

Despite improved shock sharpness, there is no reduction in the $L_{2}$ error norm measured in smooth portion of the numerical solution calculated with the second order method, as is evident in Figs. 20 and 21 which are analogous to Figs. 8 and 9. Figure 20 shows that the $L_{2}$ norm of the wall pressure error obtained with the LF2-2 scheme on locally refined grids increases by a factor of $10^{1}-10^{2}$ compared with the uniform grid results. The pressure error measured in the half of the domain closest to the body exhibits similar behavior, as is evident in Fig. 21. Note that for the second-order scheme, the error convergence rate of the local grid refinement method is less than the design order.

In contrast to the second-order method, the numerical solution error obtained with the LF-4-3 scheme is dominated by the first-order error component. Figures 22 and 23 show that the local grid refinement near the shock reduces the first-order error component generated by the LF-4-3 shock-capturing procedure, so that the design-order error component becomes dominant on the adaptive grids. Nlthough 


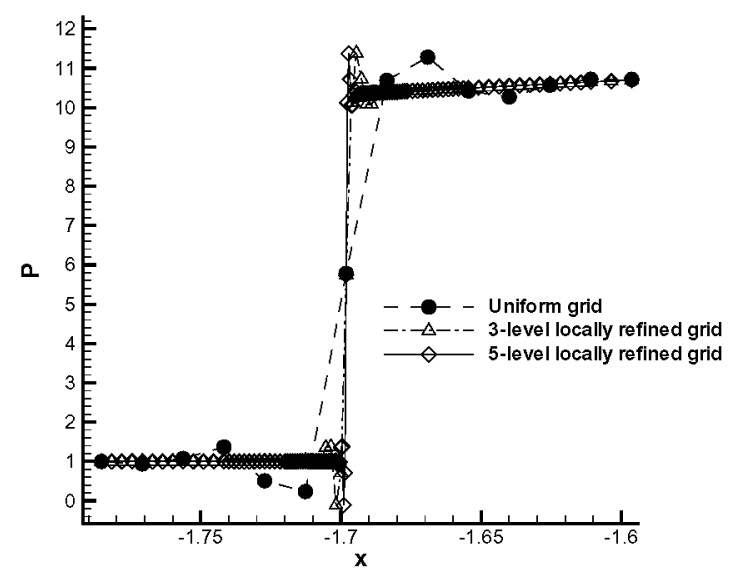

FIG. 19. Pressure distributions along line $y=0$ calculated on the $65 \times 65$ uniform and 3-, and 5-level locally refined grids.

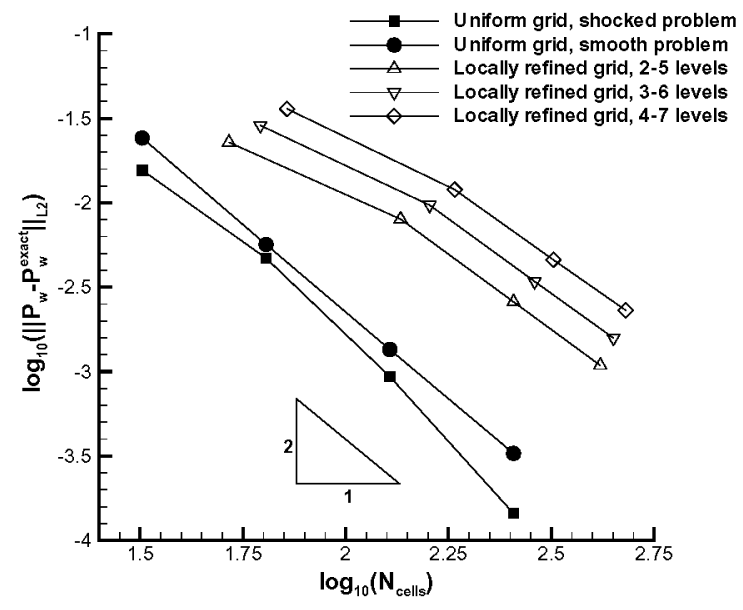

FIG. 20. Grid refinement study at $M_{\infty}=3$, showing the wall pressure error obtained with the LF-2-2 scheme on uniform and locally refined grids.

the error convergence rate is almost recovered to the design order, the $L_{2}$ norm of the wall pressure error obtained on locally refined grids becomes comparable with the uniform grid results only on the finest mesh. For coarser grids, the superiority of uniformly spaced grids is evident. The same conclusion can be drawn for the pressure error measured in the field away from the shock, except that the solution errors obtained on the locally refined and uniform grids become comparable on the coarser $129 \times 129$ grid, as one can see in Fig. 23. The numerical results obtained with both the second- and fourth-order schemes show that as the number of interfaces between coarse and fine meshes increases, the deterioration in accuracy also increases, which qualitatively corroborates the analysis presented in Section 5.2. 


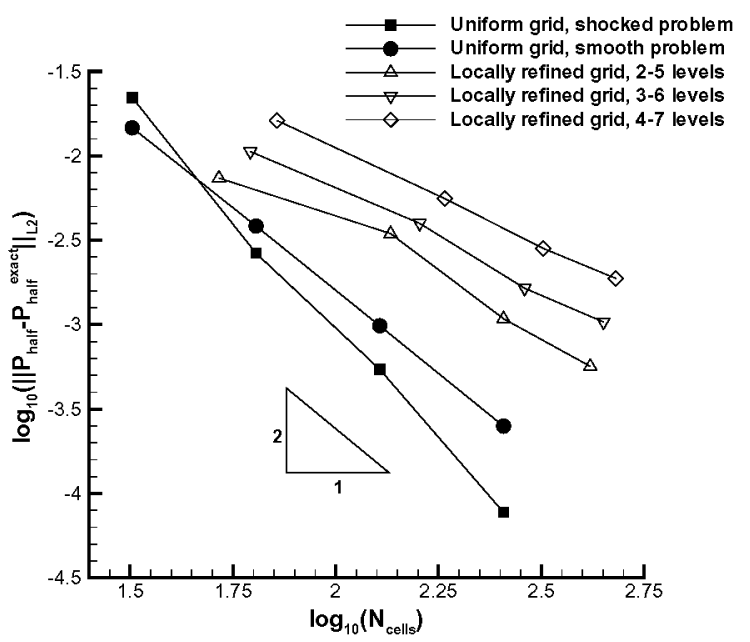

FIG. 21. Grid refinement study at $M_{\infty}=3$, showing the pressure error in the half of the domain closest to the body obtained with the LF-2-2 scheme on uniform and locally refined grids.

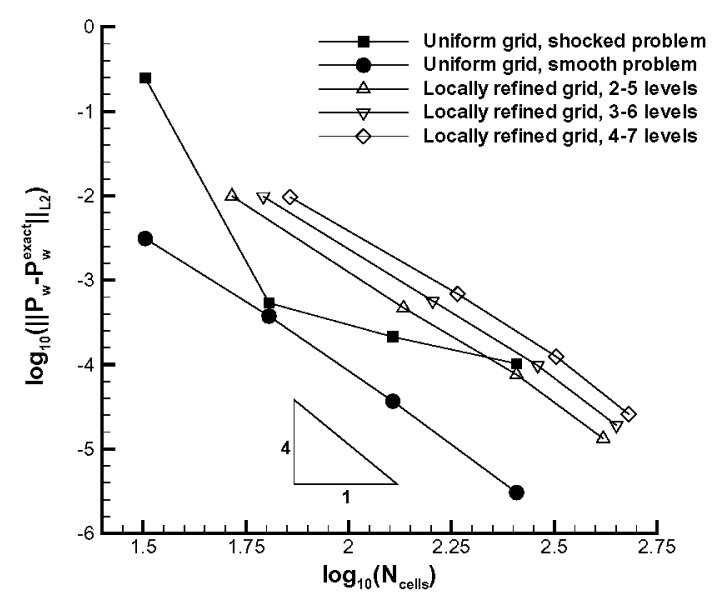

FIG. 22. Grid refinement study at $M_{\infty}=3$, showing the wall pressure error obtained with the LF-4-3 scheme on uniform and locally refined grids.

Error convergence plots of the pressure integral along the centerline calculated using the LF-2-2 and LF-4-3 schemes on the same sequences of locally refined and uniform meshes are depicted in Figs. 24 and 25, respectively. Note that the error convergence on uniform grids is monotonic, whereas the error convergence on adaptive grids exhibits nonmonotonic behavior. Figure 24 shows that the local grid refinement method based on the LF-2-2 scheme does not reduce the pressure integral error compared with the uniform grid results. Certain improvement in accuracy of the pressure integral computed with the LF-4-3 scheme can be observed on fine locally refined grids, as shown in Fig. 25. However, this gain in accuracy decreases as the background mesh is globally refined, and it practically vanishes on the finest 


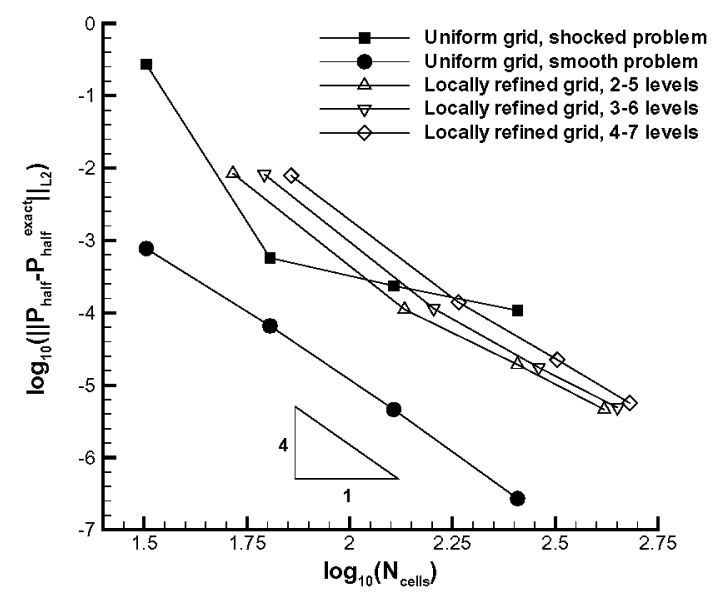

FIG. 23. Grid refinement study at $M_{\infty}=3$, showing the pressure error in the half of the domain closest to the body obtained with the LF-4-3 scheme on uniform and locally refined grids.

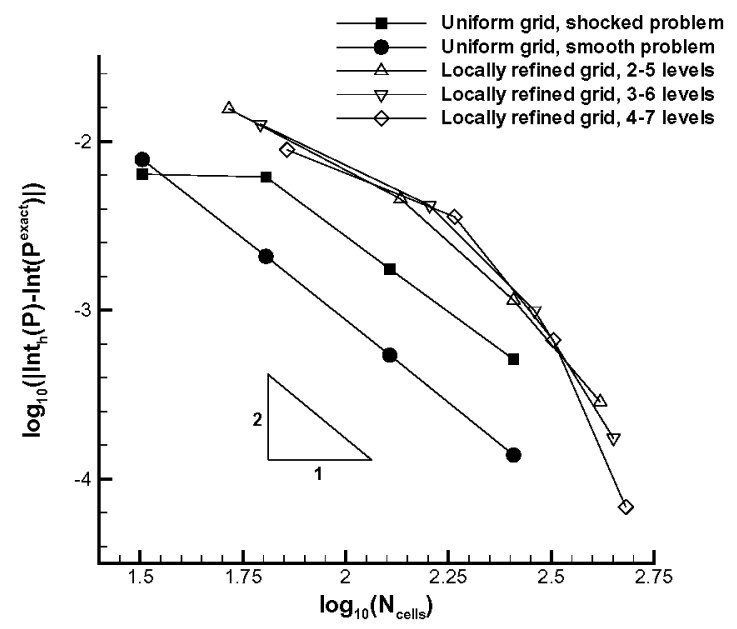

FIG. 24. Grid refinement study for the pressure integral error obtained with the LF-2-2 scheme on uniform and locally refined grids.

adaptive mesh. Although only the error in the pressure and its integral have been presented in this study, similar convergence behavior is observed for the other flow quantities.

The results presented above have been obtained under the assumption that one family of grid lines is parallel to the bow shock. However, in practical applications, generation of shock-aligned grids is a complicated problem, because the exact shock location is unknown. To gain greater insight into the error behavior when the grid is not aligned with the shock, we perform a grid refinement study on uniform grids generated by the mapping Eq.(11) with $r_{0}(\theta)=$ const. In this case, circumferential grid lines are circular arcs which are not aligned with the bow shock. Figure 26 


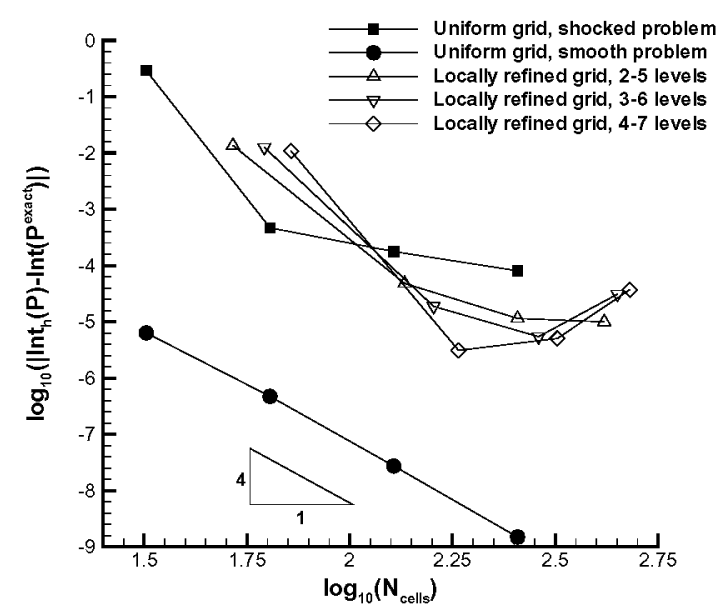

FIG. 25. Grid refinement study for the pressure integral error obtained with the LF-4-3 scheme on uniform and locally refined grids.

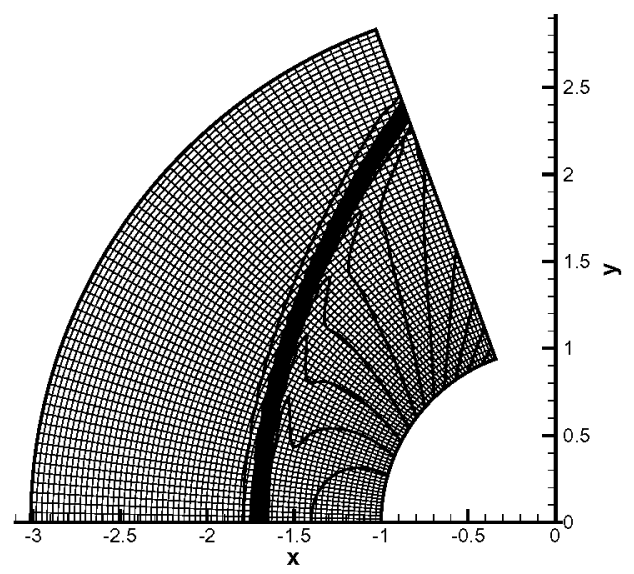

FIG. 26. Nonaligned uniform $65 \times 65$ grid and pressure contours obtained with the LF-2-2 scheme on this grid.

shows a uniform $65 \times 65$ grid and isobars of the blunt body flow calculated with the LF-2-2 scheme on this grid. Note that the maximum angle between the bow shock and circumferential grid lines, which occurs at the point where the shock crosses the outflow boundary, is about $45^{\circ}$. The $L_{2}$ norm of the wall pressure error and the pressure error norm measured in the half of the domain closest to the body surface (obtained on the nonaligned uniform grids) are compared with the shock-aligned uniform and most accurate adaptive grid results in Figs. 27 and 28, respectively. $\Lambda$ s follows from the grid refinement study, when the grid is aligned with the bow shock, the coefficient in front of the first-order error component is negligibly small, giving the appearance of a second-order convergence rate. However, this coefficient 


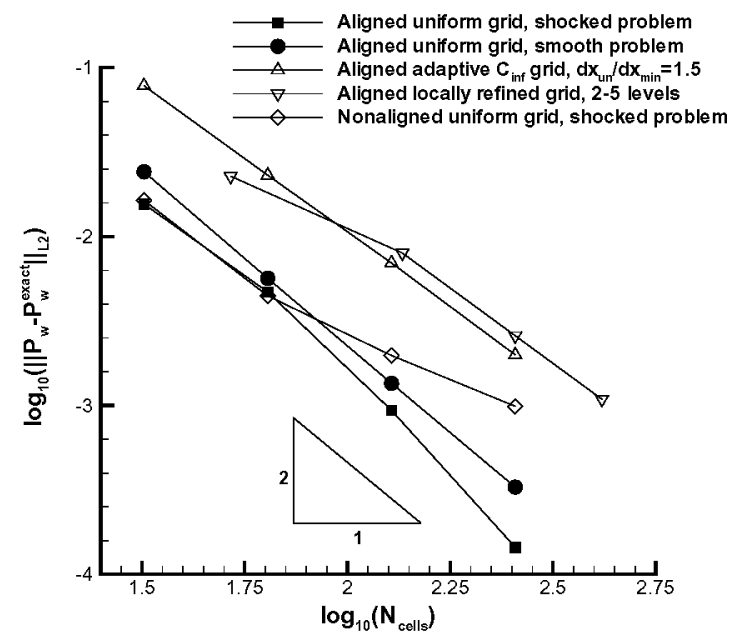

FIG. 27. Grid refinement study at $M_{\infty}=3$, showing the wall pressure error obtained with the LF-2-2 scheme on nonaligned uniform, shock-aligned uniform and adaptive grids.

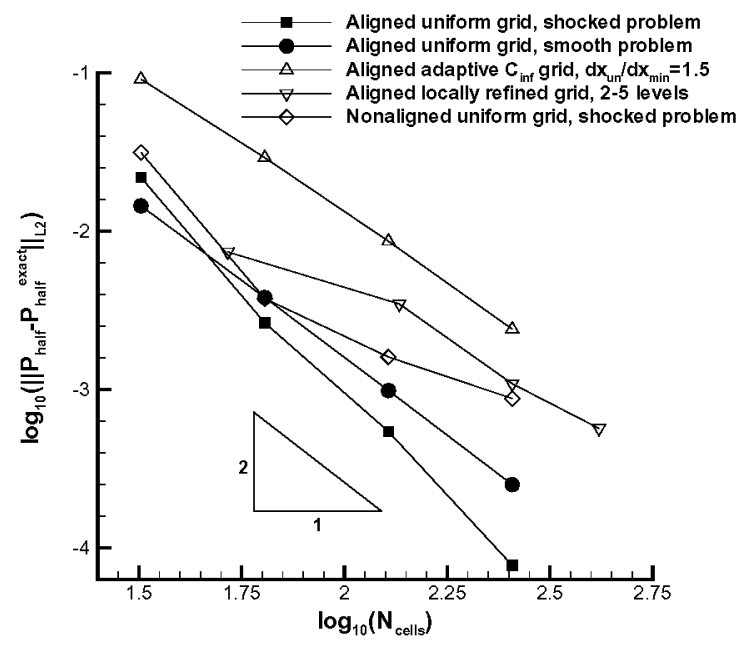

FIG. 28. Grid refinement study at $M_{\infty}=3$, showing the pressure error in the half of the domain closest to the body obtained with the LF-2-2 scheme on nonaligned uniform, shock-aligned uniform and adaptive grids.

becomes much larger on nonaligned grids that makes the first-order shock error component dominant on much coarser grids. Despite the fact that the $L_{2}$ norm of the pressure error obtained on the finest nonaligned uniform mesh is one order of magnitude less accurate than that on the corresponding shock-aligned uniform grid, the nonaligned uniform grid solution is still more accurate than the best adaptive grid results. $\Lambda$ s one can see in Figs. 27 and 28, the error convergence obtained with the LF-2-2 scheme is very similar to that calculated with the LF-4-3 scheme on shock-aligned grids. Figure 28 shows that the grid adaptation can improve the numerical solution accuracy only asymptotically. 


\section{GRID ADAPTATION CRITERION FOR CAPTURED SHOCKS}

Based on the results presented in Sections 6.1 and 6.2, we propose the following grid adaptation strategy for captured shocks. The most general error convergence behavior shown schematically in Fig. 29 is characterized by the presence of three different regions in the error convergence plot. The first region corresponds to coarse meshes such that the shock error component is of the order of $O(1)$. In this region, the grid adaptation near the shock is desirable because it reduces the most troublesome part of the error generated by the shock-capturing procedure. It should be stressed that the grid adaptation not only reduces the shock error, but also localizes the captured shock, which minimizes interaction of Gibbs oscillations with the boundary conditions and other features of the flow. This is one of the main reasons why high resolution shock-capturing schemes, such as ENO and weighted ENO schemes, provide better accuracy on coarse grids compared to high-order linear algorithms.

In the second region, which corresponds to sufficiently fine grids, the coefficient $C_{1}$ in Eq. (14) approaches its asymptotic value which, as has been shown for the LF-2-2 scheme, may be very small. For these grids, the design-order error component dominates the first-order error component, i.e. $\left\|C_{p} h^{p}\right\|>\left\|C_{1} h\right\|$. $\Lambda$ s a result, any clustering of grid points or local mesh refinement in the vicinity of the shock reduces only that part of the error which is not dominant, ultimately reducing the numerical solution accuracy in smooth portions of the solution. The main reason for such a behavior is the fact that both grid adaptation strategies are not without penalties in the solution accuracy. $\Lambda$ s follows from the truncation error analysis, for the grid redistribution method, the main source of error is due to the high-order derivatives of the metric coefficients which drastically increase on nonuniform meshes. The numerical analysis performed for the local grid refinement technique has shown that, in this case, the error accumulation occurs because of discontinuities in grid spacing and the coupling between coarse and fine meshes, herein calculated as an approximate solution of the Riemann problem. Because

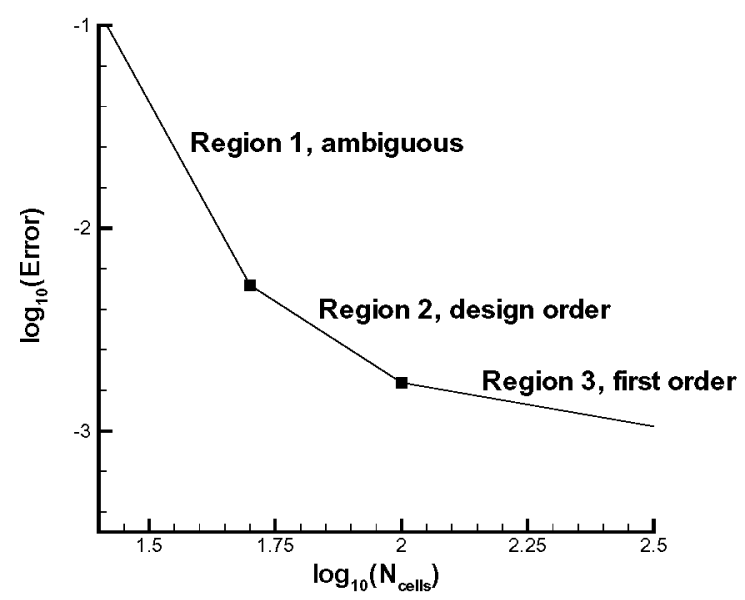

FIG. 29. Error convergence diagram for a high-order shock-capturing method. 
of these penalties in the solution accuracy, the grid adaptation increases the total error in the numerical solution if it is used in the second region.

The third region corresponds to the first-order asymptotic limit. In this region, the grid adaptation near the bow-shock would be the most efficient use of the computer resources. However, as has been shown numerically, there is a transition zone where the error convergence rate is $O(h)$, but the grid adaptation does not provide improvement in the numerical solution accuracy compared with the corresponding uniform grid results. This is because both the grid redistribution and local grid refinement methods introduce additional error in the numerical solution, caused by the grid nonuniformity. Note that the transition zone becomes larger if the computational cost is used instead of the number of grid cells in the error convergence plots.

From the above analysis it follows that the grid adaptation is desirable if the following inequality holds:

$$
\left\|C_{1} h\right\| \gg\left\|C_{p} h^{p}\right\|
$$

where the norm is measured in regions where the solution is smooth. The above inequality can be used as a grid adaptation criterion for captured discontinuities. It should be noted that the constants $C_{1}$ and $C_{p}$ depend on both the problem and the numerical scheme used. $\Lambda$ s has been mentioned earlier, the coefficient $C_{p}$ can be treated as the leading truncation error term. To our knowledge, there are no theoretical results for evaluation of the coefficient $C_{1}$. Therefore, to use the criterion (53) in practical applications, global grid refinement or coarsening is required. The global coarsening can be used if the grid is fine enough to correspond to the second and third regions in the error convergence diagram. However, if the grid is very coarse that corresponds to the first region, the coarsening cannot be used, and the grid refinement is the only way to evaluate the error convergence rate. This approach becomes quite expensive in three dimensions. From this point of view, it is very important to be able to predict a priori the error introduced by the shock-capturing procedure. This quantification together with the criterion (53) will provide guidance for grid adaptation for captured discontinuities.

It is instructive to speculate on the generality of this work, particularly on two practical questions: 1) are the conclusions and suggestions (see Fig. 29) valid for any general discontinuous flow, and 2) are the present results (second- and fourth-order Lax-Friedrichs schemes) general for any high-order numerical algorithm? Nlthough a precise answer does not presently exist to either question, we make the following conjecture: Similar qualitative results and an equivalent grid adaptation criterion (53) will be obtained for any flows containing multidimensional shocks, independent of the high-order numerical method (central difference with scalar dissipation, Roe, TVD, and ENO). This assertion is based on the observation that for any captured discontinuities, the numerical solution error obtained with any high-order shock-capturing method consists of two parts: the first-order shock error $C_{1} h$ and the design-order error component $C_{p} h^{p}$. Although the coefficients $C_{1}$ and $C_{p}$ are problem and scheme dependent, asymptotically, these coefficients do not depend on the grid spacing $h$. Consequently, for any high-order method, such that $p>1$, and any finite values $C_{1}$ and $C_{p}$, the solution error is asymptotically dominated by 
the first-order error component, corresponding to the third region in Fig. 29. If $C_{1} \ll C_{p}$, the solution error exhibits high-order convergence on sufficiently coarse grids, and corresponds to the second region in the error convergence diagram. If the grid is very coarse, such that $C_{1} h \sim O(1)$, the solution error corresponds to the first region in Fig. 29. Note, however, that the first and second regions may be very small or even nonexistent if $C_{1} \gg C_{p}$, whereas the third region always exists, regardless of the discontinuity strength and the high-order numerical algorithm used.

Although the results presented above have been obtained for steady state flows, the same conclusions can be drawn for time-dependent problems as well. $\Lambda$ n essential effect of the grid nonuniformity on the numerical solution accuracy remains one of the most important sources of error for unsteady problems with shocks. Since time-dependent flows involve both the temporal and spatial errors, additional errors caused by mesh movement and dynamical refinement/coarsening are introduced into the numerical solution. The main source of error for moving-grid methods is the inability to satisfy the geometric conservation law in the presence of moving discontinuities. One of the main problems associated with time-dependent local grid refinement methods is the need to interpolate. Note that any high-order interpolation across a strong discontinuity can cause a perceptible loss of spatial accuracy and can produce strong oscillations because of the Gibbs phenomenon. Another very important issue, particularly for unsteady problems, is a mutual influence of the temporal and spatial error components.

\section{CONCLUSIONS}

The accuracy of the adaptive grid redistribution and local grid refinement methods is examined and analyzed for captured shocks. The grid refinement study using second- and fourth-order finite difference schemes based on the Lax-Friedrichs flux vector splitting is performed to solve the supersonic inviscid flow around a circular cylinder. The numerical calculations show that if the solution error is dominated by the first-order error component, the grid adaptation near the shock increases the error convergence rate towards the design order of the numerical algorithm used. $\Lambda \mathrm{t}$ the same time, it has been shown theoretically and corroborated numerically that the design-order error component drastically increases because of the grid nonuniformity. $\Lambda$ s a result, either clustering of grid points or local grid refinement near the shock improves the numerical solution accuracy only asymptotically on very fine meshes that are not reasonable for modern computers. Furthermore, it has been found that neither grid adaptation strategy practically reduces error in the pressure integral across the shock compared with that obtained on the corresponding uniform grid. From the present analysis it follows that grid adaptation is desirable if the first-order error component measured in the smooth part of the solution is much larger than the design-order error component. This inequality can be used as a grid adaptation criterion for captured discontinuities.

\section{ACKNOWLEDGMENT}

This work was performed while the first author held a National Research Council Research Associateship Award at NASA Langley Research Center. 


\section{REFERENCES}

1. J. Casper and M. H. Carpenter, Computational considerations for the simulation of shockinduced sound, SIAM J. Sci. Comput., 19(4), 813 (1998).

2. M. H. Carpenter and J. Casper, The Accuracy of Shock Capturing in Two Spatial Dimensions, Technical Paper 97-2107 (AIAA Press, Washington, DC, 1997).

3. B. Engquist and B. Sjögreen, The convergence rate of finite difference schemes in the presence of shocks, SIAMJ. Numer. Anal., 35(6), 2464 (1998).

4. J. F. Thompson, Z. U. A. Warsi, and C. W. Mastin, Numerical Grid Generation: Foundation and Applications (North Holland, New York/Amsterdam, 1985).

5. N. K. Yamaleev, Minimization of the truncation error by grid adaptation, J. Comp. Phys., $170(2), 459(2001)$.

6. C. de Boor, Good approximation by splines with variable knots II, in Proc. of Confer. on the Numerical Solution of Differential Equations, University of Dundee, Dundee, 1978, Lecture Notes in Math., (Springer-Verlag, Berlin/Heidelberg/New York, 1973), Vol. 363, p. 12.

7. V. Pereyra and E. G. Sewell, Mesh selection for discrete solution of boundary problems in ordinary differential equations, Numer. Math., 23, 261 (1975).

8. I. Babuška and W. Rheinboldt, A-posteriori error estimates for the finite element method, Int. J. Numer. Methods Engrg. 12, 1597 (1978).

9. H. A. Dwyer, Grid adaptation for problems in fluid dynamics, AIAA J, 22 (1984).

10. D. Catherall, The adaptation of structured grids to numerical solutions for transonic flows, Int. J. Numer. Methods Engrg.: 32 (1991).

11. W. Huang and D. Sloan, A simple adaptive grid method in two dimensions, SIAM J. Sci. Comput., 15 (1994).

12. T. Sonar and E. Süli, A dual graph-norm refinement indicator for finite volume approximations of the Euler equations, Numerische Mathematik, 78, 619 (1998).

13. X. D. Zhang, D. Pelletier, J.-Y. Trepanier, and R. Camarero, Error Control and Mesh Adaptation for the Euler Equations, Technical Paper 2001-0441 (AIAA Press, Washington, DC, 2001).

14. J. Perarie, M. Vahdati, K. Morgan, and O. C. Zenkiewicz, Adaptive remeshing for compressible flow computations, J. Comp. Phys., 72, 449 (1987).

15. M. J. Berger and R. J. Leveque, Adaptive mesh refinement using wave-propagation algorithms for hyperbolic systems, SIAM J. Numer. Anal., 35(6), 2298 (1998).

16. G. P. Warren, K. Anderson, J. L. Thomas, and S. L. Krist, Grid Convergence for Adaptive Methods, Technical Paper 91-1592-CP (AIAA Press, Washington, DC, 1991).

17. M. H. Carpenter, H. L. Atkins, and D. J. Singh, Characteristic and finite-wave shock-fitting boundary conditions for Chebyshev methods, in Transition, Turbulence, and Combustion, Vol.2, edited by M. Y. Hussaini, T.B. Gatski, and T.L. Jackson, (Kluwer Academic Publishers, 1994).

18. A. Jameson, The evolution of computational methods in aerodynamics, J. Appl. Mech., 50(4b), 1052 (1983). 


\begin{tabular}{|c|c|c|c|c|}
\hline \multicolumn{3}{|c|}{ REPORT DOCUMENTATION PAGE } & & $\begin{array}{l}\text { Form Approved } \\
\text { OMB No. } 0704-0188\end{array}$ \\
\hline \multicolumn{5}{|c|}{$\begin{array}{l}\text { Public reporting burden for this collection of information is estimated to average } 1 \text { hour per response, including the time for reviewing instructions, searching existing data } \\
\text { sources, gathering and maintaining the data needed, and completing and reviewing the collection of information. Send comments regarding this burden estimate or any other } \\
\text { aspect of this collection of information, including suggestions for reducing this burden, to Washington Headquarters Services, Directorate for Information Operations and } \\
\text { Reports, } 1215 \text { Jefferson Davis Highway, Suite 1204, Arlington, VA 22202-4302, and to the Office of Management and Budget, Paperwork Reduction Project (0704-0188), } \\
\text { Washington, DC } 20503 \text {. }\end{array}$} \\
\hline 1. AGENCY USE ONLY (Leave blank) & \multicolumn{2}{|c|}{$\begin{array}{l}\text { 2. REPORT DATE } \\
\text { August } 2002\end{array}$} & \multicolumn{2}{|c|}{$\begin{array}{l}\text { 3. REPORT TYPE AND DATES COVERED } \\
\text { Technical Memorandum }\end{array}$} \\
\hline \multicolumn{3}{|c|}{$\begin{array}{l}\text { 4. TITLE AND SUBTITLE } \\
\text { On Accuracy of Adaptive Grid Methods for Captured Shocks }\end{array}$} & \multirow{2}{*}{\multicolumn{2}{|c|}{$\begin{array}{l}\text { 5. FUNDING NUMBERS } \\
706-31-11-03\end{array}$}} \\
\hline \multicolumn{3}{|c|}{$\begin{array}{l}\text { 6. AUTHOR(S) } \\
\text { Nail K. Yamaleev and Mark H. Carpenter }\end{array}$} & & \\
\hline \multicolumn{3}{|c|}{ 7. PERFORMING ORGANIZATION NAME(S) AND ADDRESS(ES) } & \multicolumn{2}{|c|}{$\begin{array}{l}\text { 8. PERFORMING ORGANIZATION } \\
\text { REPORT NUMBER }\end{array}$} \\
\hline 9. SPONSORING/MONITORING AGENCY NAME(S) AND ADDRESS(ES) & \multicolumn{2}{|c|}{$\begin{array}{l}\text { National Aeronautics and Space Administration } \\
\text { Washington, DC 20546-0001 }\end{array}$} & $\begin{array}{l}\text { 10. SPONSORING/MONITORING } \\
\text { AGENCY REPORT NUMBER }\end{array}$ & NASA/TM-2002-211415 \\
\hline \multicolumn{5}{|l|}{ 11. SUPPLEMENTARY NOTES } \\
\hline \multicolumn{3}{|c|}{$\begin{array}{l}\text { 12a. DISTRIBUTION/AVAILABILITY STATEMENT } \\
\text { Unclassified-Unlimited } \\
\text { Subject Category } 64 \quad \text { Distribution: Nonstandard } \\
\text { Availability: NASA CASI (301) 621-0390 }\end{array}$} & \multicolumn{2}{|c|}{ 12b. DISTRIBUTION CODE } \\
\hline \multicolumn{5}{|c|}{$\begin{array}{l}\text { 13. ABSTRACT (Maximum } 200 \text { words) } \\
\text { The accuracy of two grid adaptation strategies, grid redistribution and local grid refinement, is examined by } \\
\text { solving the 2-D Euler equations for the supersonic steady flow around a cylinder. Second- and fourth-order } \\
\text { linear finite difference shock-capturing schemes, based on the Lax-Friedrichs flux splitting, are used to discretize } \\
\text { the governing equations. The grid refinement study shows that for the second-order scheme, neither grid } \\
\text { adaptation strategy improves the numerical solution accuracy compared to that calculated on a uniform grid with } \\
\text { the same number of grid points. For the fourth-order scheme, the dominant first-order error component is } \\
\text { reduced by the grid adaptation, while the design-order error component drastically increases because of the grid } \\
\text { nonuniformity. As a result, both grid adaptation techniques improve the numerical solution accuracy only on the } \\
\text { coarsest mesh or on very fine grids that are seldom found in practical applications because of the computational } \\
\text { cost involved. Similar error behavior has been obtained for the pressure integral across the shock. A simple } \\
\text { analysis shows that both grid adaptation strategies are not without penalties in the numerical solution accuracy. } \\
\text { Based on these results, a new grid adaptation criterion for captured shocks is proposed. }\end{array}$} \\
\hline \multirow{2}{*}{\multicolumn{4}{|c|}{$\begin{array}{l}\text { 14. SUBJECT TERMS } \\
\text { High-order accuracy, grid adaptation, local grid refinement, grid } \\
\text { redistribution, shock capturing, finite difference scheme }\end{array}$}} & $\begin{array}{l}\text { 15. NUMBER OF PAGES } \\
42\end{array}$ \\
\hline & & & & 16. PRICE CODE \\
\hline $\begin{array}{l}\text { 17. SECURITY CLASSIFICATION } \\
\text { OF REPORT } \\
\text { Unclassified }\end{array}$ & $\begin{array}{l}\text { 18. SECURITY CLASSIFICATION } \\
\text { OF THIS PAGE } \\
\text { Unclassified }\end{array}$ & $\begin{array}{l}\text { 19. SECUR } \\
\text { OF AB } \\
\text { Uncl: }\end{array}$ & TION & $\begin{array}{l}\text { 20. LIMITATION } \\
\text { OF ABSTRACT } \\
\text { UL }\end{array}$ \\
\hline NSN 7540-01-280-5500 & & & & $\begin{array}{l}\text { Standard Form } 298 \text { (Rev. } 2-89 \\
\text { Prescribed by ANSI Std. Z-39-18 } \\
298-102\end{array}$ \\
\hline
\end{tabular}

